\title{
Pentatricopeptide repeat poly(A) binding protein from mitochondria of trypanosomes
}

Mikhail V. Mesitov ${ }^{1}$, Tian Yu ${ }^{1,2}$, Takuma Suematsu' ${ }^{1}$, Francois M. Sement ${ }^{1}$, Liye Zhang $^{3}$, Clinton Yu $\mathbf{u}^{4}$, Lan Huang ${ }^{4}$ and Inna Aphasizheva ${ }^{1, *}$

${ }^{1}$ Department of Molecular and Cell Biology, Boston University Medical Campus, Boston, MA 02118, USA;

${ }^{2}$ Bioinformatics Program, Boston University, Boston, MA 02215, USA;

${ }^{3}$ School of Life Science and Technology, ShanghaiTech University, Shanghai 201210, China;

${ }^{4}$ Department of Physiology and Biophysics, School of Medicine, University of California, Irvine, CA 92697, USA

*Corresponding author: Inna Aphasizheva, Department of Molecular and Cell Biology,

72 E. Concord St., E424, Boston, MA 02118, USA

Email: innaaf@bu.edu; Fax: 617414 1056; Phone: 617 358-3780.

https://orcid.org/0000-0003-2695-2950 


\section{Abstract}

In Trypanosoma brucei, most mitochondrial mRNAs undergo U-insertion/deletion editing, and 3'

4 adenylation and uridylation. The internal sequence changes and terminal extensions are

5 coordinated: Pre-editing addition of the short (A) tail protects the edited transcript against $3^{\prime}-5^{\prime}$

6 degradation, while post-editing A/U-tailing renders mRNA competent for ribosome recruitment.

7 Participation of a poly(A) binding protein (PABP) in coupling of editing and $3^{\prime}$ modification

8 processes has been inferred, but its identity and mechanism of action remained elusive. We

9 report identification of KPAF4, a pentatricopeptide repeat-containing PABP which sequesters

10 the A-tail and impedes exonucleolytic degradation. Conversely, KPAF4 inhibits uridylation of

11 A-tailed transcripts and, therefore, premature A/U-tailing of partially-edited mRNAs. This

12 quality check point prevents translation of incompletely edited mRNAs. Our findings also

13 implicate the RNA editing substrate binding complex (RESC) in mediating the interaction

14 between the 5' end bound pyrophosphohydrolase MERS1 and 3' end associated KPAF4 to enable

15 mRNA circularization. This event is critical for transcript stability during the editing process.

17 Key words: Trypanosoma, mitochondria, polyadenylation, RNA stability, RNA editing, PPR protein. 
The parasitic hemoflagellate Trypanosoma brucei (T. brucei) maintains a mitochondrial genome composed of catenated maxicircles and minicircles. A few 23-kb maxicircles encode 9S and 12S rRNAs, six protein-coding and 12 encrypted genes, a trans-acting MURF2-II and cis-acting CO2 guide RNAs (gRNA). Thousands of 1-kb minicircles produce gRNAs that direct Uinsertion/deletion editing of cryptic maxicircle transcripts, thus giving rise to open reading frames $^{1-3}$. Messenger and ribosomal RNA precursors are transcribed from individual promoters ${ }^{4}$ and processed by $3^{\prime}-5^{\prime}$ exonucleolytic trimming, which is followed by adenylation or uridylation,

27 respectively. Trimming is accomplished by a DSS1 $3^{\prime}-5^{\prime}$ exonuclease ${ }^{5}$ acting as subunit of the mitochondrial processome (MPsome), which also contains an RNA editing TUTase 1 (RET1) and several structural polypeptides ${ }^{6}$. Binding of the pentatricopeptide (35 amino acids) repeat (PPR) Kinetoplast Polyadenylation Factor 3 (KPAF3) to purine-rich sequences near the encoded 3' end recruits KPAP1 poly(A) polymerase and channels pre-mRNA into the adenylation/editing pathway $^{7,8}$. Conversely, rRNAs lacking KPAF3 binding sites upstream of the MPsome-generated

$333^{\prime}$ end are uridylated by RET1 TUTase ${ }^{7}$. The U-tails decorating ribosomal ${ }^{9}$ and guide RNAs ${ }^{10}$

34 reflect a mechanism in which antisense transcripts impede $3^{\prime}-5^{\prime}$ trimming thereby creating a 35 kinetic "window of opportunity" for U-tail addition", 6 . Thus, uridylation terminates rRNA and gRNA precursor degradation, but the resultant U-tails do not appreciably influence the stability

37 of mature molecules ${ }^{11,12}$. In contrast, short A-tails (20-25 nt) exert profound and opposite effects on mRNA decay depending upon the molecule's editing status. Knockdown of KPAP1 poly(A) polymerase leads to moderate upregulation of non-adenylated pre-edited mRNA, but causes a 40 rapid degradation of the same transcript edited beyond the initial few sites near the $3^{\prime}$ end ${ }^{7,8,13}$.

41 Remarkably, mRNAs containing functional coding sequence that do not require editing, referred 42 to here as unedited, also rely on KPAF3 binding and ensuing KPAP1-catalyzed A-tailing for 
43 stabilization. In massively edited (pan-edited) transcripts, sequence changes typically begin near

44 the $3^{\prime}$ end and proceed in the $3^{\prime}-5^{\prime}$ direction ${ }^{14}$. An unknown signaling mechanism monitors

45 editing status and triggers short A-tail extension into a long ( $>200 \mathrm{nt}) \mathrm{A} / \mathrm{U}-$ heteropolymer upon

46 completion of the editing process at the $5^{\prime}$ region. The A/U-tailing is accomplished by KPAP1

47 poly(A) polymerase and RET1 TUTase and requires an accessory heterodimer of PPR proteins

48 KPAF1 and KPAF2. The resultant A/U-tail does not affect the stability, but rather activates mRNA for translation by enabling binding to the small ribosomal subunit ${ }^{15}$. Thus, the temporally separated pre-editing A-tailing and post-editing A/U-tailing processes are distinct in their factor requirements and functions.

57 would leave the partially-edited transcript reliant on the short A-tail as an critical stability

59 degradation by the MPsome, or uridylation by RET1 in vivo. Indeed, these features would be essential for partially-edited mRNA stabilization and for blocking its A/U-tailing, hence premature translational activation. However, synthetic adenylated RNA represents a susceptible

62 substrate for degradation by the MPsome ${ }^{6}$ and uridylation by RET1 ${ }^{16}$ in vitro. 
moiety)) hydrolase; MERS2 PPR factor; and MERS3, a subunit lacking any motifs. The PPsome binds the $5^{\prime}$ end of a primary transcript and converts the $5^{\prime}$ triphosphate moiety incorporated at transcription initiation into a monophosphate. Intriguingly, MERS1 knockdown severely compromises edited mRNA stability without affecting 3' polyadenylation. To reconcile these observations, we hypothesized that poly(A) binding protein (PABP) may inhibit mRNA 3'-5' degradation and $3^{\prime}$ uridylation by sequestering the short A-tail. We further reasoned that PABP may interact with the PPsome at the 5' end to stabilize mRNA during the editing process. Unable to identify a canonical RRM motif-containing PABP in mitochondria, we inquired whether a

74 PPR factor capable of recognizing adenosine stretches may exist. A recognition code developed

75 for PPRs from land plants suggests that each repeat binds a single nucleotide via amino acid

76 situated in positions 5 and 35, or the last residue in helix-turn-helix motifs exceeding the

77 canonical length ${ }^{17}$. For example, a combination of threonine and asparagine in these positions,

78 respectively, recognizes an adenosine base ${ }^{18,19}$. By searching for repeats with such a pattern

79 among 38 predicted trypanosomal $\mathrm{PPRs}^{20}$, we identified a polypeptide containing five adjacent

80 repeats that would be predicted to bind as many contiguous adenosines. Termed Kinetoplast

81 Polyadenylation Factor 4 (KPAF4), this protein interacts with established components of the

82 polyadenylation and editing complexes and predominantly binds to short A-tails in vivo. KPAF4

83 knockdown downregulates A-tailed edited and unedited mRNAs, but not their A/U-tailed forms.

84 Remarkably, KPAF4 repression also permitted uridylation of A-tailed pre-edited mRNAs.

85 Specific KPAF4 binding to adenylated substrate inhibited both $3^{\prime}-5^{\prime}$ RNA degradation by the MPsome and uridylation by RET1 TUTase in vitro. Collectively, our data support a model in

87 which KPAF4 stabilizes partially and fully edited, and unedited transcripts by binding to the 88 short A-tail and enabling mRNA circularization. 
89

90

91

92

93

94

95

96

97

98

99

100

101

102

103

104

105

106

107

108

109

110

\section{Results}

\section{KPAF4 interacts with mitochondrial $m R N A$ processing complexes}

To identify a putative mitochondrial PABP, we analyzed the repeat structure and amino acids occupying positions 5 and 35 , or the last position in repeats longer than 35 residues $^{17}$, in annotated pentatricopeptide repeat-containing polypeptides from $T$. brucei $^{20}$. We searched for threonine and asparagine residues in these positions, respectively, a combination that binds an adenosine $e^{18,19}$. By considering proteins with at least four adjacent repeats, we identified a candidate $31.8 \mathrm{kDa}$ protein termed Kinetoplast Polyadenylation Factor 4 (KPAF4,

Tb927.10.10160), which consisted almost entirely of seven PPR repeats predicted with various degree of confidence. The 6-7 repeat organization is conserved among orthologous proteins in Trypanosoma and Leishmania species, while repeats R1 to R5 invariantly possess a T-N combination (Fig. 1a). Repeats 6 and 7 had the required combination shifted by one position.

Because topology prediction algorithms ranked the probability of mitochondrial targeting at 20$40 \%$, the KPAF4 localization was confirmed by subcellular fractionation. The C-terminally TAP-tagged ${ }^{21}$ KPAF4 was conditionally expressed in procyclic (insect) form of T. brucei and demonstrated to have been enriched in the mitochondrial matrix by approximately 8 -fold. Partial association with the inner membrane has also been detected (Fig. 1b).

To place the candidate protein into a functional context, KPAF4 was isolated by tandem affinity chromatography (Fig. 1c). Purifications were also conducted from a parental 29-13 cell $\operatorname{line}^{22}$ as a control and from RNase I-treated mitochondrial lysate. Final fractions were analyzed by immunoblotting for established mRNA processing factors (Fig. 1d). KPAP1, KPAF1 and 
111 KPAF3, which initiates mRNA adenylation by KPAP1 ${ }^{7}$, were readily detectable among proteins

112 co-purifying with KPAF4, but the KPAP1 and KPAF1 association appears to be RNA-

113 dependent. RNase treatment also reduced KPAF4 interactions with the PPsome (MERS1 ${ }^{12}$ ),

114 RNA editing core (REL1/2 23,24$)$ and substrate binding (GRBC1/2 12,25$)$ complexes, and KPAF1/2

115 polyadenylation factor ${ }^{15}$. Only a trace amount of RET1 TUTase ${ }^{26}$ was detected in the KPAF4

116 fraction.

Co-purification with protein complexes responsible for mRNA 5' end modification,

118 editing, A-tailing, and A/U-tailing indicates that KPAF4 likely participates in mRNA processing,

119 and that some interactions are RNA-dependent. To assess the heterogeneity and apparent

120 molecular mass of KPAF4-containing particle(s) in relation to established mRNA processing

121 complexes, mitochondrial lysates from parental and KPAF4-TAP cells were fractionated on

122 glycerol gradients. Fractions were separated on native gel and analyzed for the polyadenylation,

123 PPsome, RNA editing core (RECC), and substrate binding (RESC) complexes (Fig. 1e). In

124 agreement with previous studies, KPAP1 was detected in an unassociated form and bound to an

$125 \sim 1$ MDa complex $^{7,8}$, while KPAF4 was separated into particles of $\sim 300 \mathrm{kDa}(\mathrm{I})$ and $\sim 600 \mathrm{kDa}$

126 (II), and attached to an $\sim 1$ MDa complex (III, fractions 6 and 7). Notably, RNase pre-treatment

127 of mitochondrial lysate mostly eliminated the 1MDa KPAF4 complex III but left smaller

128 particles unaffected. The PPsome and RNA editing substrate binding complex (RESC) co-

129 fractionated as an $\sim 1 \mathrm{MDa}$ particle that closely resembles complex III, while the RECC migrated

130 as a distinct $\sim 800 \mathrm{kDa}$ particle. Collectively, these results demonstrate that KPAF4 is a

131 mitochondrial pentatricopeptide repeat factor engaged with at least three macromolecular

132 complexes. The largest KPAF4-contanining complex III with an apparent molecular mass of 
$133 \sim 1 \mathrm{MDa}$ closely resembles a ribonucleoprotein assembly that encompasses PPsome, RESC and 134 polyadenylation complexes ${ }^{4,25}$.

\section{RESC tethers PPsome and polyadenylation complexes}

137 To gain a higher-resolution view of the KPAF4 interactome, the normalized spectral abundance 138 factors (NSAF) ${ }^{27}$ were derived from LC-MS/MS analysis of tandem affinity purified complexes 139 and used to build an interaction network (Fig. 2a). Polyadenylation enzyme KPAP1 and factors KPAF1, KPAF2 and KPAF3 were analyzed along with the MERS1 subunit of the PPsome ${ }^{7,8,25}$.

141 The strongest predicted KPAF4 interactions included those with a hypothetical protein lacking 142 any discernible motifs, Tb927.3.2670, and with the polyadenylation mediating module (PAMC) 143 of the RNA editing substrate binding complex ${ }^{25}$. KPAP1 and KPAF3 also featured prominently 144 among KPAF4-associated proteins. Interestingly, relatively high levels of MRP1 and MRP2 145 were detected in KPAF4 preparation (Supplementary Table 1). A subject of extensive

146 investigation, heterotetramer MRP1/2 RNA chaperone displays RNA annealing activity in vitro,

147 but its definitive function remains undetermined ${ }^{28-31}$. The ternary interaction between KPAF4,

$148 \mathrm{~Tb} 927.3 .2670$, and the MRP1/2 RNA chaperone complex was verified by cross-tagging of

149 MRP2 and the hypothetical protein. Mass spectrometry analysis of samples purified from

150 RNase-treated extracts indicated that interactions between KPAP1 poly(A) polymerase, and

151 KPAF1-2 and KPAF3 polyadenylation factors are sufficiently stable to withstand a two-step

152 purification, but nonetheless depend on an RNA component (Supplementary Table 1). KPAF4-

153 MRP1/2-Tb927.3.2670 co-purification, on the other hand, was unaffected by RNase treatment.

154 Importantly, the network predicted that the RESC complex may facilitate co-complex

155 interactions between the PPsome and KPAF4. 
To corroborate the interaction network inferences, we investigated the proximity of

157 KPAF4, polyadenylation, RESC and PPsome complexes by in vivo biotinylation (BioID ${ }^{32}$ ),

158 which has an estimated $10 \mathrm{~nm}$ labeling range ${ }^{33}$. KPAP1, GRBC2, MERS1, and KPAF4 were

159 conditionally expressed as C-terminal fusions with BirA* biotin ligase and biotinylation was

160 induced for 24 hours. Labeled proteins were purified under denaturing conditions and analyzed

161 by LC-MS/MS (Fig. 2b and Supplementary Table 2). The BioID experiments placed KPAP1 in

162 proximity to the KPAF2 polyadenylation factor, subunits P3 and P4 of the polyadenylation

163 mediator module (PAMC), and Tb927.3.2670. Surprisingly, MRP2 emerged as the major

164 biotinylated protein in cells expressing KPAP1, MERS1 and GRBC2 fusions with BirA*. In

165 aggregate, the co-purification, apparent molecular mass assessment of KPAF4 complexes and in

166 vivo proximity studies suggest that KPAF4 interacts with the mitochondrial polyadenylation and

167 RNA editing substrate binding complexes. It seems plausible that GRBC and REMC modules of

168 the latter mediate the co-complex interaction between KPAF4 and the PPsome.

KPAF4 is essential for parasite growth and for maintaining a subset of mitochondrial mRNAs

171 The potential role of KPAF4 in mitochondrial RNA processing and parasite viability was

172 examined in the insect (procyclic) form of T. brucei. Inducible RNAi knockdown efficiently

173 downregulated KPAF4 mRNA (Fig. 3a) and triggered a cell growth inhibition phenotype after

174 approximately 24 hours, indicating that KPAF4 is essential for normal cellular function (Fig. 3b).

175 Quantitative RT-PCR of RNA samples isolated at 55 hours post-RNAi induction demonstrated

176 divergent effects of KPAF4 knockdown on mRNA abundance. Downregulation of moderately

177 edited (CYB and MURF2), and some pan-edited (RPS12, ND3 and CO3) mRNAs was

178 accompanied by upregulation of their respective pre-edited forms. The transcript-specific effects 
were also apparent for unedited transcripts that either remained relatively steady (CO1 and ND5) or increased (ND1, MURF1 and ND4). Finally, mitochondrial ribosomal RNAs remained virtually unaffected, which indicates an mRNA-specific KPAF4 function (Fig. 3c). We next tested whether these effects may have been caused by KPAF4 RNAi-induced changes in steadystate levels of known processing factors. Immunoblotting analysis showed that KPAP1 poly(A) polymerase was downregulated by approximately $50 \%$ in KPAF4 RNAi background while other tested enzymes and RNA binding proteins remained unchanged (Fig. 3d).

\section{KPAF4 knockdown differentially affects mRNAs depending on their editing status}

Albeit instructive, the global changes in relative abundance provide limited information about $3^{\prime}$ modifications and their correlation with mRNA editing status. To assess whether moderate KPAP1 decline in the KPAF4 RNAi background (Fig. 3d) may have compromised mRNA adenylation, we performed time-resolved analysis of pan-edited mRNAs. The representative example, RPS12 mRNA, constitutes a single domain in which editing initiates close to the polyadenylation site and traverses the entire transcript in a $3^{\prime}-5^{\prime}$ hierarchical order as directed by multiple overlapping gRNAs ${ }^{14}$. Samples from KPAF3 knockdown cells were also separated by high resolution gel electrophoresis to typify impeded mRNA adenylation and accelerated decay ${ }^{7}$. Northern blotting with probes for pre-edited, partially-edited ( $\sim 70 \%$ completed, $5^{\prime}$ region not edited) and fully-edited variants also distinguishes non-adenylated, A-tailed and A/U-tailed mRNAs (Fig. 4a, Supplementary Fig.1). Upon KPAF3 repression, an initial loss of the short Atail ( 0 - 48 hours of RNAi induction), was followed by rapid mRNA degradation. In contrast, KPAF4 knockdown led to lengthening and, in agreement with qRT-PCR results (Fig. 3c), to a moderate increase in pre-edited mRNA abundance. While partially-edited mRNA patterns 
202

203

204

205

206

207

208

209

210

211

212

213

214

215

216

mirrored the loss of the pre-edited form in KPAF3 knockdown, similar populations remained virtually unchanged in length and abundance with progression of KPAF4 RNAi. The fully-edited transcripts displayed a more complex pattern in KPAF4-depleted cells: The A-tailed form declined while the A/U-tailed form remained unaffected. To investigate the unexpected lengthening of pre-edited RNAs in KPAF4 knockdown cells, the 3' extensions were amplified, cloned and sequenced. In agreement with a previous report for the parental 29-13 strain of $T$. brucei $^{8}$, in 96 clones obtained from mock-induced KPAF4 RNAi short A-tails varied within 2025 nt range (not shown). Remarkably, A-tails not only persisted in KPAF4 knockdown, but in $\sim 30 \%$ of clones were extended into oligo(U) stretches (Fig. $4 \mathrm{~b}$ and Supplementary Table 3).

These results demonstrate that, unlike KPAF3, KPAF4 is not required for pre-edited mRNA stabilization and adenylation, but it may prevent spurious uridylation of A-tailed transcripts. The disposition apparently changes with progression of editing in KPAF4 RNAi background: Fullyedited short A-tailed mRNAs decline while A/U-tailed transcript remain unaffected. It follows that KPAF4 may stabilize fully-edited A-tailed mRNA but is not required for its A/U-tailing upon completion of editing.

Extending northern blotting analysis to another pan-edited mRNA encoding subunit A6 of ATP synthase showed a similar response to KPAF4 depletion: substantial lengthening and upregulation of pre-edited RNA accompanied by downregulation of the edited A-tailed form (Fig. 4c). In moderately-edited CYB mRNA, where 34 uridines are inserted close to the 5' end, the pre-edited form was upregulated while the edited variant behaved like pan-edited mRNAs (Fig. 4d). In unedited mRNAs, such as CO1 and ND1, short A-tailed populations also declined while A/U-tailed ND1 increased more than 10-fold (Fig. 4e, Supplementary Fig.1). Finally, the lack of detectable impact on ribosomal RNAs (Fig. 4f), which are also produced from maxicircle 
225

226

227

228

229

230

231

232

233

234

235

236

237

238

239

240

241

242

243

244

245

246

247

and normally uridylated, confirmed that KPAF4 is an mRNA-specific factor. Minicircle-derived gRNAs were either unaffected, such as gA6(14), or moderately upregulated, as in the case of gCO3(147) (Fig. 4g). The latter effect correlates with a loss of corresponding edited CO3 mRNA (Fig. 3c), as reported for genetic knockdowns that eliminate edited mRNAs ${ }^{11}$. Thus, the outcomes of KPAF4 knockdown are consistent with its hypothetical function as a poly(A) binding protein: stabilization of A-tailed edited mRNA that is no longer bound by KPAF3 ${ }^{7}$, but not yet channeled into the post-editing A/U-tailing reaction ${ }^{15}$.

\section{KPAF4 inhibits $\mathrm{mRNA}$ uridylation in vivo}

In pre-edited mRNA, the mature 3' end is produced by MPsome-catalyzed trimming and KPAF3-stimulated adenylation? ${ }^{7}$. The short A-tailed mRNA is then somehow protected from $3^{\prime}-5^{\prime}$ degradation during editing, and from KPAF1/2-stimulated A/U-tailing ${ }^{15}$ until the editing process is completed ${ }^{8}$. Although conventional cloning and sequencing provided preliminary indication that KPAF4 may inhibit uridylation of short A-tailed mRNA (Fig. 4b), this technique's limitations prevented analysis of longer A-rich extensions. To obtain a comprehensive view of mRNA 3' termini in a KPAF4 RNAi background, we combined mRNA circularization with single molecule real time sequencing (SMRT, PacBio platform) and deep sequencing-bysynthesis (Illumina platform) to characterize short and long tails in pre-edited, edited and unedited mRNAs, and ribosomal RNAs (Supplementary Fig. 2). RNAs expected have only short tails, such as pre-edited RPS12, A6 and CYB transcripts, we sequenced on Illumina platform while their edited forms known to have both short and long tails were sequenced with PacBio platform $^{34}$. Unedited CO1 mRNA, expected to have short and long tails, and U-tailed rRNA were sequenced on both platforms. The long-range SMRT sequencing of A/U-tails revealed an 
approximately 50:50 A/U ratio in edited and unedited mRNAs (Fig. 5D, which is somewhat different than previously calculated 70:30 ratio ${ }^{15}$. The molecular cloning of $3^{\prime}$ extensions in the original report likely caused the observed differences with this study. Length classification of short 3' extensions into $10 \mathrm{nt}$ bins (Fig. 5a), and long ones into $10 \mathrm{nt}$ and $50 \mathrm{nt}$ bins (Fig. 5b), exposed higher heterogeneity and general shortening of short A-tails in pre-edited transcripts upon KPAF4 RNAi induction for 72 hours. In contrast, corresponding pan-edited RPS12 and A6 mRNAs, and unedited CO1, possessed a higher percentage of tails in the 150-250 nt range, which encompasses the bulk of A/U-tailed mRNAs. The lack of effect on ribosomal RNAs further establishes KPAF4 as an mRNA-specific factor. We also noticed that the A/U-tail length distribution derived from real-time PacBio sequencing was consistent with the apparent length determined by northern blotting (Fig. 4a), as sequences longer than $400 \mathrm{nt}$ were detected (Fig. 5d). Plotting of nucleotide frequencies from short range sequencing also confirmed A-tail shortening accompanied by uridylation in pre-edited RPS12 and A6 mRNAs (Fig. 5c). As indicated by distribution of adenosine and uridine residues in long tails, lack of KPAF4 leads to earlier emergence of U-rich structures (Fig. 5d). Noteworthy, the high-fidelity short-range Illumina sequencing confirmed rRNA's uridylated status, while the real-time PacBio platform was uninformative for short $3^{\prime}$ extensions. In conclusion, $3^{\prime}$ tail sequencing on two independent platforms connected the loss of KPAF4 with the spurious addition of U-tails to adenylated mRNAs, and with general stimulation of A/U-tail synthesis.

\section{KPAF4 binds A-tails in vivo}

To establish KPAF4 in vivo binding specificity, we applied UV-crosslinking of live cells, and two-step affinity purification of TAP-6His-tagged polypeptide followed by deep sequencing 
271 (CLAP-Seq, Fig. 6a). We note that maxicircle genes encode rRNAs, and unedited and pre-edited

272 mRNAs, which are typically separated by short non-coding regions. Since most genes are

273 transcribed from dedicated promoters as 3' extended precursors, the mature mRNA 3' ends

274 produced by $3^{\prime}-5^{\prime}$ trimming often extend into $5^{\prime}$ regions of downstream genes ${ }^{4}$. In KPAF4

275 CLAP-Seq, $\sim 40 \times 10^{6}$ reads originated from maxicircle transcripts and edited mRNAs, while only

$276 \sim 9 \times 10^{6}$ reads mapped to the minicircles constituting more than $90 \%$ of kinetoplast DNA ${ }^{35,36}$.

277 Mapping of CLAP-Seq reads to the maxicircle revealed a preference for 3' ends of pre-edited

278 and unedited transcripts encoded on both DNA strands. Conversely, most reads derived from

279 abundant ribosomal RNAs clustered within 9S rRNA (Fig. 6b). At the mRNA level, plotting a

280 nucleotide frequency within reads that partially mapped to unedited and edited transcripts

281 demonstrated a strong bias toward adenosine residues at the $3^{\prime}$ region (Fig. 6c). A composite read

282 mapping and nucleotide frequency plot calculated for unedited and fully-edited mRNAs with the

283 termination codon set as zero further demonstrates KPAF4's preferential binding near

284 polyadenylation sites and to short A-tails, but not long A/U-tails (Fig. 6d). Interestingly, pure A-

285 tracks accounted for approximately $0.5 \%\left(2 \times 10^{5}\right)$ of all unmapped KPAF4-CLAP reads while

286 sequences ending with more than 30 As constituted $33 \%\left(1.5 \times 10^{6}\right)$ of all reads mapped to

287 mitochondrial mRNAs. Mapping statistics for tail sequencing and KPAF4-CLAP are provided in

288 Supplementary Table 4.

To test whether in vivo oligo(A) binding specificity is conferred by amino acid residues

290 occupying positions 5 and 35 or the last residue in KPAF4 repeats, we introduced T5N and

291 N35/36D substitutions into all seven PPRs (Fig. 1a). The expression levels of mutated variant

292 (KPAF4-Mut) and KPAF4-WT were virtually identical (Supplementary Fig. 3a) and produced

293 negligible growth phenotype (Supplementary Fig. 3b) while LC-MS/MS analysis demonstrated a 
294

295

296

297

similar composition of respective affinity purified samples (Supplementary Table 5). However, in CLAP-Seq experiments KPAF4-Mut showed markedly reduced crosslinking efficiency (Supplementary Fig. 3c) and low background coverage of mitochondrial transcripts (Supplementary Fig. 3d).

KPAF4 knockdown leads to uridylation and upregulation of pre-edited mRNA, but also causes concurrent decay of the A-tailed edited form (Fig. 4a, b and e). To elucidate the connection between the mRNA's editing status and KPAF4-dependent stabilization, we compared read coverage between individual pre-edited and fully-edited mRNAs; nucleotide frequencies were also included to detect non-encoded $3^{\prime}$ additions (Fig. 6e). A consistent pattern in pan-edited RPS12 and A6 mRNA showed that KPAF4 preferentially binds to the 5' and 3' regions, including A-tails, in pre-edited transcripts, but is confined to $3^{\prime}$ regions in fully-edited mRNAs. In moderately-edited CYB mRNA, the editing-dependent re-distribution of reads was similar, except for adenosine enrichment at the pre-edited $5^{\prime}$ end, a likely outcome of reads mapping to the $3^{\prime}$ end of the closely-spaced upstream CO3 mRNA. These observations suggest that KPAF4 binds to both $5^{\prime}$ and $3^{\prime}$ termini in pre-edited transcripts, possibly leading to mRNA circularization. Furthermore, sequence changes introduced by editing and/or remodeling of ribonucleoprotein complexes during the editing process, apparently displace KPAF4 from 5' regions, where the editing process comes to completion. The circularization suggested by KPAF4 binding to both mRNA ends (Fig. 6e) and cross-talk between 3' end-bound KPAF4 and $5^{\prime}$ end-bound PPsome (Fig. 2) may be critical for inhibiting $3^{\prime}-5^{\prime}$ degradation ${ }^{7}$. These observations may provide a mechanistic basis for the rapid decay of edited mRNA in MERS1 knockdown ${ }^{12}$. MERS1 pyrophosphohydrolase binds to the $5^{\prime}$ terminus and removes pyrophosphate from the first nucleotide incorporated by transcription, but the mechanism of 
317 mRNA stabilization by MERS1 remains unclear ${ }^{4}$. If circularization indeed takes place, we 318 reasoned that MERS1 would also be expected to bind the 3' end and/or A-tails. Mapping of

319 MERS1-CLAP reads to the same transcripts exposed the KPAF4-like re-distribution of MERS1

320 binding sites from the $5^{\prime}$ end in pre-edited to both $5^{\prime}$ and $3^{\prime}$ termini including A-tails in edited

321 mRNAs (Fig. 6f). In sum, in vivo crosslinking experiments indicate that pan-editing events

322 eliminate KPAF4 binding sites in pre-edited transcripts and confine this factor to the $3^{\prime}$ region

323 and short A-tail. These events are likely responsible for KPAF4-mediated protection of A-tailed

324 edited mRNA against $3^{\prime}-5^{\prime}$ degradation by the mitochondrial processome.

KPAF4 inhibits uridylation and degradation of adenylated RNAs in vitro

327 Recombinant KPAP1 poly(A) polymerase activity is intrinsically limited to adding 20-25

328 adenosines $^{8}$, while RET1 TUTase processively polymerizes hundreds of uridines in vitro ${ }^{26}$.

329 Although both enzymes lack a pronounced RNA specificity, RET1 is most efficient on substrates

330 terminating with several $\mathrm{Us}^{16}$. Likewise, uridylated RNAs represent the preferred substrate for

331 the MPsome in vivo and in vitro ${ }^{6}$. It follows that a factor responsible for blocking uridylation and

332 stabilization of adenylated mRNA would specifically bind A-tailed RNA and interfere with

333 RET1 and MPsome activities. To investigate whether KPAF4 possesses such properties, we have

334 established an in vitro reconstitution system composed of affinity purified KPAF4 and DSS1

335 exonuclease complexes, and recombinant KPAP1 and RET1 enzymes. We used synthetic $81 \mathrm{nt}$

336 RNA resembling a 3' region of edited RPS12 mRNA, and RNAs extended with either 20 As or

$33720 \mathrm{Us}$, in parallel experiments with purified KPAF4-WT and KPAF4-Mut (Fig. 7a and

338 Supplementary Table 5). 
In an electrophoretic mobility shift assay (EMSA), only adenylated RNA formed a single

340

341

342

343

344

345

346

347

348

349

350

351

352

353

354

distinct ribonucleoprotein complex commensurate with increasing KPAF4-WT concentration

(Fig. 7b). Conversely, KPAF4-Mut failed to bind any of the substrates within the protein

concentration range afforded by the assay (Fig. 7c). In enzymatic reactions with no-tail RNA,

RET1 and KPAP1 produced patterns like those reported for generic RNA substrates: distributive addition of $\sim 15$ As and processive polymerization of hundreds of Us, respectively (Fig. $7 \mathrm{~d},{ }^{8,37}$ ).

In reactions containing a mixture of both enzymes, the extension patterns were dominated by

RET1 activity. Uridylated RNA was efficiently utilized by RET1 but proved to be a poor substrate for KPAP1. In contrast to no-tail and U-tailed RNA, KPAP1 inhibited processive uridylation of the A-tailed substrate by RET1 TUTase. Unlike KPAF3, which dramatically stimulates KPAP1 activity on any tested RNA ${ }^{7}$, KPAF4 did not produce noticeable effects on either RET1 or KPAP1 activities with no-tail or U-tail RNA. However, KPAF4 inhibited processive uridylation of A-tailed RNA by RET1 TUTase, and this effect was further enhanced by KPAP1. Together, these results demonstrate that KPAF4 specifically recognizes adenylated RNAs and inhibits their uridylation by RET1 TUTase. Importantly, KPAF4's inhibitory effect on uridylation is enhanced by KPAP1 poly(A) polymerase.

The MPsome-catalyzed 3'-5' degradation represents a major processing pathway for rRNA, mRNA, and gRNA precursors, and is also responsible for decay of mature molecules ${ }^{6,7}$. While KPAF3 has been shown to protect any RNA against degradation by the MPsome in vitro ${ }^{7}$, KPAF4 binding properties and knockdown outcomes suggest that it may preferentially inhibit degradation of adenylated RNAs. To test this hypothesis, we reconstituted mRNA degradation with affinity-purified MPsome and the same 5' radiolabeled substrates used in binding and 3' extension assays. Reactions were performed for a fixed duration in the presence of increasing 
362 KPAF4 concentrations (Fig. 7e, left panels), or a time course was followed in the presence of a

363 constant KPAF4 amount (Fig. 7e, right panels). Quantitation of KPAF4 concentration- or time-

364 dependent decrease of input substrate demonstrated that the MPsome degrades no-tail or

365 uridylated RNAs irrespective of KPAF4 presence. However, KPAF4 specifically inhibits

366 hydrolysis of adenylated RNA by the MPsome (Supplementary Fig. 4). These experiments

367 illustrate that KPAF4 in vitro properties are consistent with the expected functions of a poly(A)

368 binding protein in: 1) Recognizing the A-tail; 2) Protecting adenylated mRNA against premature

369 uridylation by RET1 TUTase; and 3) Inhibiting degradation of adenylated mRNA by the

370 MPsome. 


\section{Discussion}

374 Extensive studies of the unicellular parasite Trypanosoma brucei revealed physical interactions

375 and functional coupling between protein complexes that convert cryptic mitochondrial transcripts

376 into translation-competent mRNAs. Among many transformations, constrained adenylation by

377 KPAP1 poly(A) polymerase is critical for edited and unedited mRNA stability ${ }^{8,13}$. Addition of

378 20-25 adenosines is stimulated by KPAF3 polyadenylation factor, which is recruited to pre-

379 edited mRNA, but is then displaced by editing events ${ }^{7}$. Thus, transcripts edited beyond a few

380 initial sites depend on the short A-tail for protection against destruction by the mitochondrial

381 processome. Although $3^{\prime}-5^{\prime}$ exonucleolytic degradation is the main decay mechanism, mRNA

382 stabilization also requires binding of PPsome subunit MERS1 to the 5' end. Finally, post-editing

383 A/U-tailing involving RET1 TUTase activates ribosome recruitment and translation, but this

384 reaction is somehow blocked during the editing process to avoid synthesis of aberrant proteins

385 from mRNA lacking an open reading frame ${ }^{15}$. To reconcile these observations, we envisaged that

386 a trans-acting factor may recognize a nascent A-tail to enable an interaction between protein

387 complexes occupying 5' and $3^{\prime}$ mRNA termini. Consequentially, this would increase resistance

388 to degradation and uridylation. In this study, we identified the pentatricopeptide repeat-

389 containing factor KPAF4 as essential for normal parasite growth and demonstrated its role in

390 recognizing 3' A-tails, preventing mRNA uridylation by RET1, and inhibiting 3'-5' degradation

391 of adenylated mRNAs by the MPsome.

PPR proteins are defined by arrays of approximately 35-amino acid helix-turn-helix

393 motifs $^{38}$, each recognizing a single nucleotide via amino acid side chains occupying cardinal

394 positions 5 and $35^{17}$. Bioinformatic analysis of trypanosomal PPRs identified KPAF4 as a factor

395 potentially capable of binding five consecutive adenosines, and, therefore, a candidate for a 
mitochondrial poly(A) binding protein. Biochemical fractionation, immunochemical and proteomics experiments demonstrate that KPAF4 interacts with polyadenylation and RNA editing substrate binding (RESC) complexes. In agreement with an established architecture of the RESC, KPAF4 contacts are mostly confined to the polyadenylation mediator module (PAMC), which has been defined as a docking site for the polyadenylation complex ${ }^{25}$. A binding platform for RNA editing substrates and products ${ }^{3,39}$, RESC also recruits enzymatic RNA editing core complex and, importantly for mRNA stabilization, the 5' end-bound PPsome ${ }^{4}$. Therefore, it seems plausible that RESC-mediated interaction network provides a physical basis for functional coupling among 5' pyrophosphate removal by MERS1, KPAP1-catalyzed 3' adenylation, and internal U-insertion/deletion editing. To that end, in vivo crosslinking identified 3 ' termini and 406 short A-tails as KPAF4 primary recognition sites, but also detected binding events in the 5' region. KPAF4 CLAP-Seq coverage displayed an instructive correlation with the editing status: The $3^{\prime}$ termini including A-tails were occupied in all tested mRNA types (pre-edited, edited and unedited), while the $5^{\prime}$ regions were bound chiefly in pre-edited mRNAs. Remarkably, these

410 patterns were mirrored by editing-dependent re-distribution of MERS1 binding sites.

411 Collectively, interaction networks, proximity studies, and identification of in vivo binding sites

412 point toward circularization as the major mRNA surveillance and stabilization event. In this

413 scenario, only adenylated pre-mRNA proceeds through the editing pathway while being

414 protected by KPAF4-bound short A-tail from an assault by the MPsome, which degrades RNA ${ }^{7}$, 415 and from A/U-tailing, which activates translation ${ }^{15}$. 
419 specific loss of A-tailed molecules, but minimal impact on post-editing A/U-tailing reaction,

420 which is accomplished by KPAP1, RET1 and KPAF1/2 polyadenylation factors. It seems likely

421 that the A/U-tailed mRNA no longer depends on KPAF4-mediated stabilization mechanism. The

422 argument can be extended to suggest that completion of editing results in KPAF4 displacement

423 from the short A-tail and/or loss of interaction with the $5^{\prime}$ end. These events would enable RET1

424 access and trigger A/U-tailing. The presence of a protein "sensor" monitoring RNA editing

425 completion has been suggested ${ }^{20}$, but further studies are required to decipher a signaling

426 mechanism. The KPAF4 stabilizing role is somewhat similar to PPR10 in maize chloroplasts,

427 which defines mRNA 3' end by binding to a specific site and impeding $3^{\prime}-5^{\prime}$ degradation ${ }^{40}$. The

428 distinction in lies in post-trimming addition of the KPAP4 binding platform.

430 similar function to that of an RRM domain, a universal fold of canonical poly(A) binding

431 proteins $^{41}$. Although the recognition mechanisms are likely to be different, KPAF4 properties are

432 well aligned with a paradigm for PPR repeats as sequence-specific readers and modulators of

433 diverse enzymatic activities. The latter effects can be stimulatory, as typified by KPAF $1 / 2^{15}$ and

$434 \mathrm{KPAF}^{7}$, or inhibitory, like those conferred by KPAF4. 


\section{Methods}

\section{Parasite maintenance, $R N A$ i, protein expression and $R N A$ analysis}

438 Plasmids for RNAi knockdowns were generated by cloning an 500-bp gene fragment into 439 p2T7-177 vector for tetracycline-inducible expression ${ }^{42}$. Linearized constructs were transfected 440 into a procyclic 29-13 T. brucei $\operatorname{strain}^{22}$. For inducible protein expression, full-length genes were 441 cloned into pLew-MHTAP vector ${ }^{43}$. For BioID experiments, full-length genes were cloned into 442 the same vector with the C-terminal TAP tag replaced by a mutated BirA* ligase from E. coli ${ }^{32}$.

\section{Biochemical analysis}

444 RNAi, mitochondrial isolation, glycerol gradient, native gel, total RNA isolation, northern and 445 western blotting, qRT-PCR, and tandem affinity purification were performed as described in ${ }^{44}$.

446 The change in relative abundance was calculated based on qRT-PCR, or northern blotting, data 447 assuming the ratio between analyzed transcripts and control RNAs in mock-induced cells as 1 or $448100 \%$, respectively. BioID purifications were performed from crude mitochondrial fractions, as 449 described in the Appendix.

\section{Protein identification by LC-MS/MS}

451 Affinity-purified complexes were sequentially digested with LysC peptidase and trypsin. LC452 MS/MS was carried out by nanoflow reversed phase liquid chromatography (RPLC) (Eksigent, 453 CA) coupled on-line to a Linear Ion Trap (LTQ)-Orbitrap mass spectrometer (Thermo-Electron 454 Corp). A cycle of full FT scan mass spectrum (m/z 350-1800, resolution of 60,000 at m/z 400) was 455 followed by $10 \mathrm{MS} / \mathrm{MS}$ spectra acquired in the linear ion trap with normalized collision energy 456 (setting of 35\%). Following automated data extraction, resultant peak lists for each LC-MS/MS 457 experiment were submitted to Protein Prospector (UCSF) for database searching similarly as 
458 described ${ }^{45}$. Each project was searched against a normal form concatenated with the random form

459 of the T. brucei database (http://tritrypdb.org/tritrypdb/).

\section{Sequencing of RNA 3' extensions}

461 Total RNA $(10 \mu \mathrm{g})$ was circularized with T4 RNA ligase $1^{8}$, digested with RNase R (Epicenter)

462 to remove linear RNA, and termini were amplified with gene-specific primers listed in

463 Supplementary Information. Two biological replicates of long range Single Molecule Real-Time

464 (SMRT) sequencing of 0.2-4 kb fragments was performed on a PacBio RS II system (Pacific

465 Biosciences). Highly similar data sets (Pearson correlation coefficient 0.89) were combined for

466 final analysis. A single round of short range sequencing was performed on a MiSeq instrument in

467300 nt mode.

468 Crosslinking-affinity purification and sequencing (CLAP-Seq)

469 UV-crosslinking, affinity purification and RNA-Seq library preparation from KPAF4- and

470 MERS1-bound RNA fragments have been performed as described ${ }^{44}$, with modifications and

471 details of bioinformatics analysis outlined in the Appendix.

472 In vitro reconstitution

473 Edited RPS12 mRNA fragments were prepared by in vitro transcription and 5' radiolabeled.

474 No-tail: GGGTGGTGGTTTTGTTGATTTACCCGGTGTAAAGTATTATACACGTATTGU

475 AAGUUAGAUUUAGAUAUAAGAUAUGUUUUU

476 A-tail: GGGTGGTGGTTTTGTTGATTTACCCGGTGTAAAGTATTATACACGTATT

477 GUAAGUUAGAUUUAGAUAUAAGAUAUGUUUUUAAAAAAAAAAAAAAAAAAAA 
480 MPsome assays were carried out in $20 \mu 1$ reaction containing $50 \mathrm{mM}$ Tris-HCl, $\mathrm{pH}$ 8.0, $1 \mathrm{mM}$

481 DTT, 2 units/ $\mu$ RNaseOut ribonuclease inhibitor (Life Technologies), $0.1 \mathrm{mM} \mathrm{MgCl}_{2}, 20,000$

$482 \mathrm{cpm}$ of $5^{\prime}$-labeled RNA, $2 \mu 1$ of TAP-purified DSS1 fraction and $50 \mathrm{nM}$ of KPAF4. The reaction

483 was pre-incubated at $30^{\circ} \mathrm{C}$ for $20 \mathrm{~min}$, and started with the addition of DSS1. Aliquots were

484 separated on $10 \%$ polyacrylamide/8M urea denaturing gel. Phosphor images were acquired with

485 Typhoon FLA 7000 (GE Healthcare).

486 Data availability.

487 All data generated or analyzed during this study are included in this article (and its

488 Supplementary Information files). KPAF4 CLAP-Seq and tail sequencing data were deposited 489 into the Sequence Read Archive (https://www.ncbi.nlm.nih.gov/sra) under accession number 490 PRJNA477550. Sequence analysis scripts are available at tinyurl.com/y7x2txkh. 


\section{Acknowledgments}

497 We thank members of our laboratories and Ruslan Aphasizhev for discussions and technical

498 advice. This research was supported by NIH grant AI113157 to I.A.

499

500 Author contributions

501 M.M., T.S., C.Y. and I.A. carried out the experiments and contributed to discussion. T.Y., L.Z.

502 and L.H. analyzed data, and developed analytical tools and contributed to discussion. I.A.

503 designed experiments and wrote the paper. I.A. serves as the guarantor. 


\section{Figure legends}

506

Fig. 1 Repeat organization, subcellular localization and complex association of KPAF4.

508

509

510

(a) Schematic repeat organization of Kinetoplast Polyadenylation Factor 4 from Trypanosoma brucei $(\mathrm{Tb})$ and Leishmania infantum (Li). Repeat boundaries were determined using the TPRpred online tool (https://toolkit.tuebingen.mpg.de/\#/tools/tprpred) and adjusted according to Cheng et $\mathrm{al}^{17}$. Amino acids in positions 5 and $35 /$ last potentially involved in adenosine recognition are indicated in separate columns.

(b) Mitochondrial targeting of KPAF4-TAP fusion protein. Crude mitochondrial fraction was isolated by hypotonic lysis and differential centrifugation (crude mito), and further purified by Renografin density gradient (pure mito). The latter preparation was extracted under conditions that separate matrix from membrane-bound proteins ${ }^{44}$. Protein profiles were visualized by Sypro Ruby staining and KPAF4-TAP was detected with an antibody against the calmodulin binding peptide. The mitochondrial enrichment was calculated by quantitative western blotting vs. total protein loading.

(c) Tandem affinity purification of KPAF4. Final fraction was separated on 8-16\% SDS gel and stained with Sypro Ruby.

(d) KPAF4 co-purification with mRNA processing complexes. Fractions purified from parental cell line (beads, no tagged protein expressed), and mock and RNase-treated mitochondrial extracts were subjected to immunoblotting with antibodies against MERS1 NUDIX hydrolase (PPsome subunit), KPAP1 poly(A) polymerase, KPAF1 and KPAF3 polyadenylation factors, and GRBC1/2 (RNA editing substrate binding complex, RESC) and 
RET1 TUTase (MPsome). Tagged KPAF4 was detected with antibody against calmodulin binding peptide. RNA editing core complex (RECC) was detected by self-adenylation of REL1 and REL2 RNA ligases in the presence of $\left[\alpha-{ }^{32} \mathrm{P}\right] \mathrm{ATP}$.

530

(e) Crude mitochondrial fraction was extracted with detergent and soluble contents were separated for 5 hours at 178,000 g in a 10-30\% glycerol gradient. Each fraction was resolved on $3-12 \%$ Bis-Tris native gel. Positions of native protein standards are denoted by arrows. KPAP1, KPAF4-TAP, MERS1 and GRBC1/2 were visualized by immunoblotting. REL1 and REL2 RNA ligases were detected by self-adenylation. Thyroglobulin (19S) and bacterial

Fig. 2 KPAF4 interactions and proximity networks.

(a) Model of the interactions between KPAF4, KPAP1 poly(A) polymerase, KPAF1-2 and KPAF3 polyadenylation factors, RNA editing substrate binding complex (RESC), and MRP1/2 RNA chaperones. KPAP1, KPAF1, KPAF2, KPAF3, KPAF4, MRP2, MERS1 and Tb927.3.2670 proteins (encircled in red) were affinity purified from mitochondrial lysates. The network was generated in Cytoscape software from bait-prey pairs in which the prey protein was identified by five or more unique peptides. The edge thickness correlates with normalized spectral abundance factor (NSAF) values ranging from $2.9 \times 10^{-3}$ to $4.4 \times 10^{-5}$ (Supplementary Table 1). Edges between tightly bound RESC modules (GRBC, REMC and

(b) KPAF4 proximity network. Spectral counts derived from BioID experiments with KPAP1, 
build a proximity network. The edge thickness correlates with normalized spectral abundance factor (NSAF) values ranging from $2.9 \times 10^{-3}$ to $2.6 \times 10^{-5}$ (Supplementary Table 2). All purifications were performed in parallel under uniform conditions.

Fig. 3. KPAF4 repression effects on cell growth and polyadenylation complex.

555

556

557

558

559

560

561

562

563

564

565

566

567

568

569

570

571

572

(a) Northern blotting analysis of KPAF4 mRNA downregulation by inducible RNAi.

(b) Growth kinetics of procyclic parasite cultures after mock treatment and KPAF4 RNAi induction with tetracycline.

(c) Quantitative real-time RT-PCR analysis of RNAi-targeted KPAF4 mRNA, and mitochondrial rRNAs and mRNAs. The assay distinguishes edited and corresponding preedited transcripts, and unedited mRNAs. RNA levels were normalized to $\beta$-tubulin mRNA. RNAi was induced for 55 hours. Error bars represent the standard deviation from at least three biological replicates. The thick line at " 1 " reflects no change in relative abundance; bars above or below represent an increase or decrease, respectively. P, pre-edited mRNA; E, edited mRNA.

(d) Cell lysates prepared at indicated time points of KPAF4 RNAi induction were sequentially probed by quantitative immunoblotting with antigen-purified antibodies against KPAP1, KPAF1, KPAF3, GRBC1/2, and monoclonal antibodies against RET1 TUTase. Samples were normalized by protein loading.

\section{Fig. 4 Divergent effects of KPAF4 knockdown on mitochondrial RNAs.}

(a) Northern blotting of pre-edited (Pre-E), partially-edited (Part-E), and fully-edited RPS12 mRNA variants. Total RNA was separated on a 5\% polyacrylamide/8M urea gel and 
sequentially hybridized with radiolabeled single-stranded DNA probes. Zero-time point: mock-induced RNAi cell line. Cytosolic 5.8S rRNA was used as loading control. Parent, RNA from parental 29-13 cell line; (dT), RNA was hybridized with 20-mer oligo(dT) and treated with RNase H to show positions of non-adenylated molecules in parental cell line. Pre-edited RNA length increase in KPAF4 RNAi is shown by brackets.

(b) Alignment of representative RPS12 mRNA 3' ends in KPAF4 RNAi cells. RNA termini were amplified by cRT-PCR, cloned and sequenced ${ }^{8}$. A fragment of $3^{\prime}$ untranslated region, short A-tail and U-extensions are indicated.

(c) Northern blotting of pan-edited A6 mRNA. Total RNA was separated on a 1.7\% agarose/formaldehyde gel and sequentially hybridized with oligonucleotide probes for preedited and fully-edited sequences. Loading control: cytosolic 18S rRNA.

(d) Northern blotting of moderately-edited $c y b$ mRNA. Total RNA was separated on a 1.7\% agarose/formaldehyde gel and hybridized with oligonucleotide probes for pre-edited and fully-edited sequences. Loading control: cytosolic 18S rRNA.

(e) Northern blotting of unedited CO1 and ND1 mRNAs. Total RNA was separated on a 1.7\% agarose/formaldehyde gel and sequentially hybridized with oligonucleotide probes. Loading control: cytosolic $18 \mathrm{~S}$ rRNA.

(f) Northern blotting of mitochondrial ribosomal RNAs. Total RNA was separated on a 5\% polyacrylamide/8M urea gels and hybridized with oligonucleotide probes. Loading control: cytosolic 5.8S rRNA.

(g) Guide RNA northern blotting. Total RNA was separated on a 10\% polyacrylamide/8M urea gel and hybridized with oligonucleotide probes specific for gA6(14) and gCO3(147). Mitochondrially-localized tRNA ${ }^{\text {Cys }}$ served as loading control. 
Fig. 5 Sequencing of mRNA and rRNA 3' extensions in KPAF4 RNAi background.

598

599

600

601

602

603

604

605

606

607

608

609

610

611

612

613

614

615

616

617

618

(a) Length distribution of short mRNA and 12S rRNA tails. Non-encoded 3' end extensions (MiSeq instrument, Illumina, single biological replicate) were individually binned into 10-nt length groups. Mock-induced and RNAi datasets, indicated by blue and red bars, respectively, represent percentage of the total number of reads.

(b) Length distribution of long mRNA and 12S rRNA tails. Non-encoded 3' end extensions (PacBio RS II instrument, two biological replicates) were individually binned into 10-nt length groups before $100 \mathrm{nt}$, and in 50-nt groups thereafter. Mock-induced and RNAi datasets are indicated by blue and red bars, respectively, that represent percentage of the total number of reads.

(c) Positional nucleotide frequencies in short mRNAs and 12S rRNA tails. A nucleotide percentage was calculated for each position that contained at least $5 \%$ of the total extracted sequences. The nucleotide bases are color-coded as indicated.

(d) Positional nucleotide frequencies in long mRNA and 12S rRNA tails. A nucleotide percentage was calculated for each position that contained at least $5 \%$ of the total extracted sequences. The nucleotide bases are color-coded as indicated.

\section{Fig. 6 Distribution of KPAF4 in vivo binding sites between pre-edited and edited mRNAs.}

(a) Isolation of in vivo KPAF4-RNA crosslinks. Modified TAP-tagged fusion protein was purified by tandem affinity pulldown from UV-irradiated $(+)$ or mock-treated $(-)$ parasites. The second purification step was performed under fully-denaturing conditions and resultant fractions were subjected to partial on-beads RNase I digestion and radiolabeling. Upon 
separation on SDS PAGE, RNA-protein crosslinks were transferred onto nitrocellulose protein crosslinks were detected by exposure to phosphor storage screen (right panel). RNA from areas indicated by brackets was sequenced. Representative of three biological replicates

623 is shown.

624 (b) KPAF4 in vivo binding sites. Crosslinked fragments were mapped to the maxicircle's genecontaining region. Annotated mitochondrial transcripts encoded on major and minor strands are indicated by blue and red arrows, respectively.

627

(c) Position-specific nucleotide frequency in partially mapped KPAF4 CLAP-Seq reads. In reads selected by partial mapping to maxicircle and edited mRNAs, the unmapped 3 ' segments were considered as tail sequences. The nucleotide frequency was calculated for each position beginning from the $3^{\prime}$ end.

(d) Aggregate KPAF4 mRNA binding pattern. Read coverage is represented by the grey area, and the nucleotides in $3^{\prime}$ extensions are color-coded at their projected positions.

633 (e) KPAF4 binding to representative pan-edited (RPS12, A6) and moderately edited (CYB) mRNAs. Read coverage profiles were created for matching pre-edited and fully edited mRNA. Read coverage is represented by the grey area, and the unmapped nucleotides in $3^{\prime}$ extensions are color-coded at their projected positions. The mRNA is highlighted with a rose bar in the context of adjacent maxicircle sequences.

638 (f) MERS1 binding to representative pan-edited (RPS12, A6), and moderately edited (CYB) mRNAs. Graphs were created as in panel $(E)$. 
641 Fig. 7 KPAF4-bound adenylated RNA is partially resistant to uridylation and degradation

642

643

644

645

646

647

648

649

650

651

652

653

654

655

656

657

658

659

660

661

in vitro.

(a) Western blotting of affinity purified KPAF4-WT and KPAF4-Mut samples. Protein samples were purified from mitochondrial fraction by rapid affinity pulldown with IgG-coated magnetic beads. KPAF4 polypeptides were detected with an antibody against the calmodulin binding peptide.

(b) Electrophoretic mobility shift assay with KPAF4-WT. Increasing amounts of affinitypurified KPAF4 were incubated with 5' radiolabeled RNAs and separated on 3-12\% native PAGE.

(c) Electrophoretic mobility shift assay with KPAF4-Mut was performed as in (b).

(d) RNA adenylation and uridylation. KPAP1, RET1, or in combination, were incubated with 5' radiolabeled RNA and ATP, UTP, or ATP/UTP mix, respectively, in the absence or presence of KPAF4. Recombinant enzymes were purified from bacteria as described ${ }^{8,46}$. Reactions were terminated at indicated time intervals and products were resolved on $10 \%$ polyacrylamide/ $8 \mathrm{M}$ urea gel.

(e) RNA degradation. The same RNA substrates as in $(d)$ were incubated with increasing (left panels) or constant (right panels) concentrations of KPAF4 in the presence or absence of the MPsome. Reactions were terminated at indicated time intervals and products were resolved on a $10 \%$ polyacrylamide/ $8 \mathrm{M}$ urea gel. Input RNA and final degradation products of 4-5 nt (FP) are shown. 


\section{References}

1. Aphasizhev, R. \& Aphasizheva, I. Mitochondrial RNA Processing In Trypanosomes. Research in Microbiology 162, 655-663 (2011).

2. Aphasizhev, R. \& Aphasizheva, I. Mitochondrial RNA editing in trypanosomes: Small RNAs in control. Biochimie 100, 125-131 (2014).

3. Read, L.K., Lukes, J. \& Hashimi, H. Trypanosome RNA editing: the complexity of getting $\mathrm{U}$ in and taking $\mathrm{U}$ out. Wiley Interdiscip Rev RNA 7, 33-51 (2016).

4. Sement FM, S.T., Zhang L, Yu T, Huang L, Aphasizheva I, Aphasizhev R. Transcription initiation defines kinetoplast RNA boundaries. BioRxiv MS ID\#:

BIORXIV/2018/350256(2018).

5. Mattiacio, J.L. \& Read, L.K. Roles for TbDSS-1 in RNA surveillance and decay of maturation by-products from the 12S rRNA locus. Nucleic Acids Res 36, 319-329 (2008).

6. Suematsu, T. et al. Antisense Transcripts Delimit Exonucleolytic Activity of the Mitochondrial 3' Processome to Generate Guide RNAs. Mol Cell 61, 364-78 (2016).

7. Zhang, L. et al. PPR polyadenylation factor defines mitochondrial mRNA identity and stability in trypanosomes. EMBO J 36, 2435-2454 (2017).

8. Etheridge, R.D., Aphasizheva, I., Gershon, P.D. \& Aphasizhev, R. 3' adenylation determines mRNA abundance and monitors completion of RNA editing in T. brucei mitochondria. EMBO J 27, 1596-1608 (2008).

9. Adler, B.K., Harris, M.E., Bertrand, K.I. \& Hajduk, S.L. Modification of Trypanosoma brucei mitochondrial rRNA by posttranscriptional 3' polyuridine tail formation. Mol. Cell. Biol 11, 5878-5884 (1991).

10. Blum, B. \& Simpson, L. Guide RNAs in kinetoplastid mitochondria have a nonencoded 3' oligo-(U) tail involved in recognition of the pre-edited region. Cell 62, 391-397 (1990).

11. Aphasizheva, I. \& Aphasizhev, R. RET1-catalyzed Uridylylation Shapes the Mitochondrial Transcriptome in Trypanosoma brucei. Molecular and Cellular Biology 30, 1555-1567 (2010).

12. Weng, J. et al. Guide RNA-Binding Complex from Mitochondria of Trypanosomatids. Molecular Cell 32, 198-209 (2008).

13. Kao, C.Y. \& Read, L.K. Opposing effects of polyadenylation on the stability of edited and unedited mitochondrial RNAs in Trypanosoma brucei. Mol. Cell Biol 25, 1634-1644 (2005).

14. Maslov, D.A. \& Simpson, L. The polarity of editing within a multiple gRNA-mediated domain is due to formation of anchors for upstream gRNAs by downstream editing. Cell 70, 459-467 (1992).

15. Aphasizheva, I., Maslov, D., Wang, X., Huang, L. \& Aphasizhev, R. Pentatricopeptide Repeat Proteins Stimulate mRNA Adenylation/Uridylation to Activate Mitochondrial Translation in Trypanosomes. Molecular Cell 42, 106-117 (2011).

16. Aphasizheva, I., Aphasizhev, R. \& Simpson, L. RNA-editing terminal uridylyl transferase 1: identification of functional domains by mutational analysis. J. Biol. Chem 279, 24123-24130 (2004).

17. Cheng, S. et al. Redefining the structural motifs that determine RNA binding and RNA editing by pentatricopeptide repeat proteins in land plants. Plant $J \mathbf{8 5}, 532-47$ (2016). 
18. Shen, C. et al. Structural basis for specific single-stranded RNA recognition by designer pentatricopeptide repeat proteins. Nat Commun 7, 11285 (2016).

19. Coquille, S. et al. An artificial PPR scaffold for programmable RNA recognition. Nat Commun 5, 5729 (2014).

20. Aphasizhev, R. \& Aphasizheva, I. Emerging roles of PPR proteins in trypanosomes: Switches, blocks, and triggers. RNA. Biol 10, 1495-1500 (2013).

21. Puig, O. et al. The tandem affinity purification (TAP) method: a general procedure of protein complex purification. Methods 24, 218-229 (2001).

22. Wirtz, E., Leal, S., Ochatt, C. \& Cross, G.A. A tightly regulated inducible expression system for conditional gene knock-outs and dominant-negative genetics in Trypanosoma brucei. Mol. Biochem. Parasitol 99, 89-101 (1999).

23. Aphasizhev, R. et al. Isolation of a U-insertion/deletion editing complex from Leishmania tarentolae mitochondria. EMBO J 22, 913-924 (2003).

24. Schnaufer, A. et al. Separate Insertion and Deletion Subcomplexes of the Trypanosoma brucei RNA Editing Complex. Mol Cell 12, 307-319 (2003).

25. Aphasizheva, I. et al. RNA binding and core complexes constitute the Uinsertion/deletion editosome. Mol. Cell Biol 34, 4329-4342 (2014).

26. Aphasizhev, R. et al. Trypanosome Mitochondrial 3' Terminal Uridylyl Transferase (TUTase): The Key Enzyme in U-insertion/deletion RNA Editing. Cell 108, 637-648 (2002).

27. Neilson, K.A., Keighley, T., Pascovici, D., Cooke, B. \& Haynes, P.A. Label-free quantitative shotgun proteomics using normalized spectral abundance factors. Methods Mol Biol 1002, 205-22 (2013).

28. Koller, J. et al. Trypanosoma brucei gBP21. An arginine-rich mitochondrial protein that binds to guide RNA with high affinity. J. Biol. Chem 272, 3749-3757 (1997).

29. Aphasizhev, R., Aphasizheva, I., Nelson, R.E. \& Simpson, L. A 100-kD complex of two RNA-binding proteins from mitochondria of Leishmania tarentolae catalyzes RNA annealing and interacts with several RNA editing components. RNA 9, 62-76 (2003).

30. Schumacher, M.A., Karamooz, E., Zikova, A., Trantirek, L. \& Lukes, J. Crystal structures of T. brucei MRP1/MRP2 guide-RNA binding complex reveal RNA matchmaking mechanism. Cell 126, 701-711 (2006).

31. Muller, U.F., Lambert, L. \& Goringer, H.U. Annealing of RNA editing substrates facilitated by guide RNA-binding protein gBP21. EMBO J 20, 1394-1404 (2001).

32. Roux, K.J., Kim, D.I., Raida, M. \& Burke, B. A promiscuous biotin ligase fusion protein identifies proximal and interacting proteins in mammalian cells. $J$ Cell Biol 196, 801-10 (2012).

33. Kim, D.I. et al. Probing nuclear pore complex architecture with proximity-dependent biotinylation. Proc Natl Acad Sci U S A 111, E2453-61 (2014).

34. Sharon, D., Tilgner, H., Grubert, F. \& Snyder, M. A single-molecule long-read survey of the human transcriptome. Nat Biotechnol 31, 1009-14 (2013).

35. Ochsenreiter, T., Cipriano, M. \& Hajduk, S.L. KISS: the kinetoplastid RNA editing sequence search tool. RNA 13, 1-4 (2007).

36. Hong, M. \& Simpson, L. Genomic organization of Trypanosoma brucei kinetoplast DNA minicircles. Protist 154, 265-279 (2003). 
37. Rajappa-Titu, L. et al. RNA Editing TUTase 1: structural foundation of substrate recognition, complex interactions and drug targeting. Nucleic Acids Res 44, 10862-10878 (2016).

38. Small, I.D. \& Peeters, N. The PPR motif - a TPR-related motif prevalent in plant organellar proteins. Trends Biochem Sci 25, 46-47 (2000).

39. Aphasizheva, I. \& Aphasizhev, R. U-Insertion/Deletion mRNA-Editing Holoenzyme: Definition in Sight. Trends Parasitol 13, 1078-1083 (2015).

40. Prikryl, J., Rojas, M., Schuster, G. \& Barkan, A. Mechanism of RNA stabilization and translational activation by a pentatricopeptide repeat protein. Proc. Natl. Acad. Sci. U.S. A 108, 415-420 (2010).

41. Goss, D.J. \& Kleiman, F.E. Poly(A) binding proteins: are they all created equal? Wiley Interdiscip Rev RNA 4, 167-79 (2013).

42. Wickstead, B., Ersfeld, K. \& Gull, K. Targeting of a tetracycline-inducible expression system to the transcriptionally silent minichromosomes of Trypanosoma brucei. Mol. Biochem. Parasitol 125, 211-216 (2002).

43. Jensen, B.C. et al. Characterization of protein kinase CK2 from Trypanosoma brucei. Mol Biochem Parasitol 151, 28-40 (2007).

44. Aphasizheva, I., Zhang, L. \& Aphasizhev, R. Investigating RNA editing factors from trypanosome mitochondria. Methods 107, 23-33 (2016).

45. Fang, L. et al. Mapping the protein interaction network of the human COP9 signalosome complex using a label-free QTAX strategy. Mol. Cell Proteomics 11, 138-147 (2012).

46. Aphasizhev, R. \& Aphasizheva, I. RNA Editing Uridylyltransferases of Trypanosomatids. Methods in Enzymology 424, 51-67 (2007). 
TbKPAF4 (Tb927.10.10160, 288 aa)

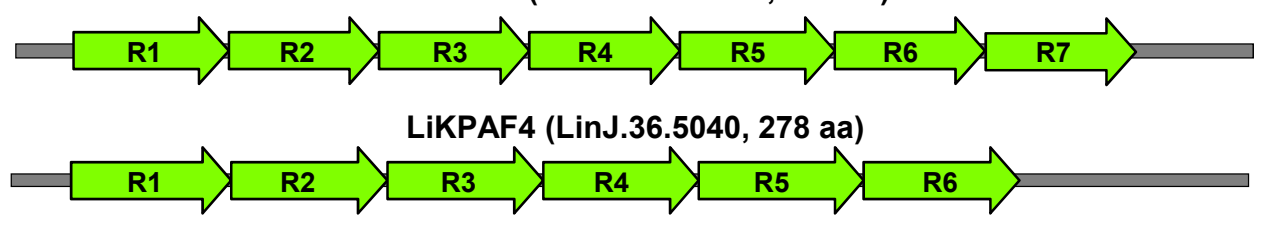

TbKPAF4, positions in the repeat: 5 35/last \begin{tabular}{ll|l|l|l|}
\hline R1 14 SRWLTDELVALSKAGNWEAAISTFLQLQQANIVQSN 49 & $\mathrm{~T}$ & $\mathbf{N}=\mathrm{A}$
\end{tabular} \begin{tabular}{|l|l|l|l|}
\hline R2 50 VFHYTTVISACGRAGKWEAAMSIFDQMTKNEVKPN 84 & $\mathrm{~T}$ & $\mathbf{N}=\mathbf{A}$
\end{tabular} R3 85 VYTYTAVINACASAEKADVALRMFAHMRLADVPPN $119 \quad$ T $\quad$ N $=\mathbf{A}$ \begin{tabular}{ll|l|l|l|}
\hline R4 120 VQTMTALVNACARSGEWERAIKILRDCEELFVAPN 154 & $\mathrm{~T}$ & $\mathbf{N}=\mathbf{A}$
\end{tabular} R5 155 VFTYTAAMDGCRRGGVWKPAVDLLNEMRDPTRVRPN 190 T $\quad$ N = A

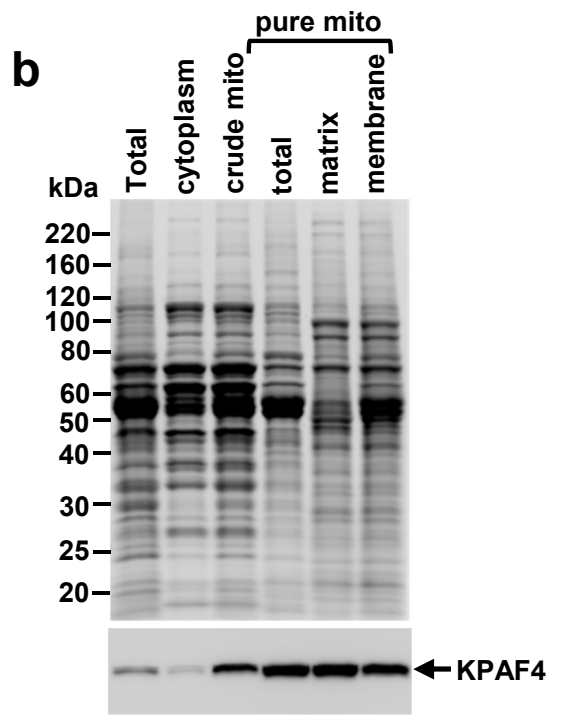

e

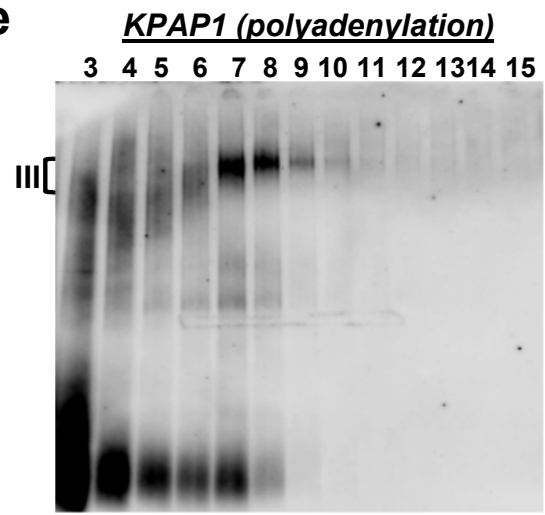

MERS1 (PPsome)

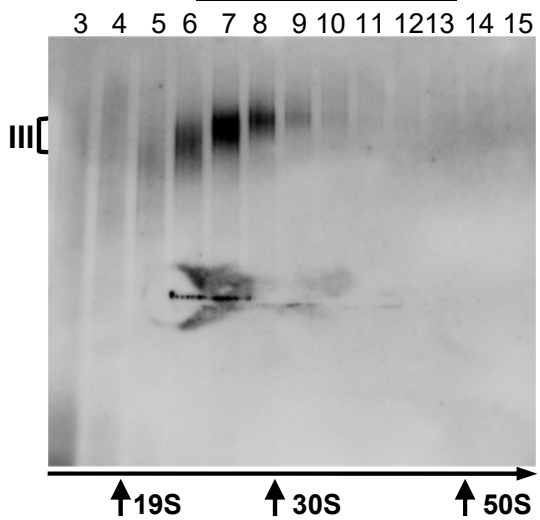

C

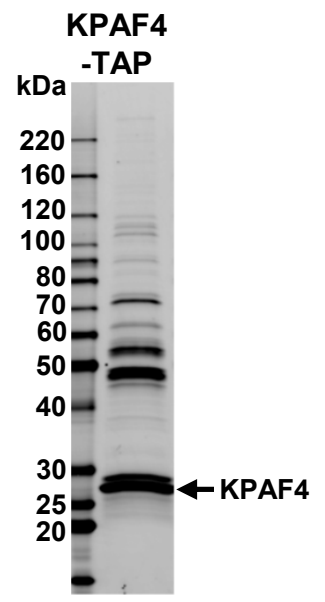

KPAF4-TAP (mock)

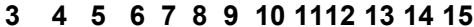

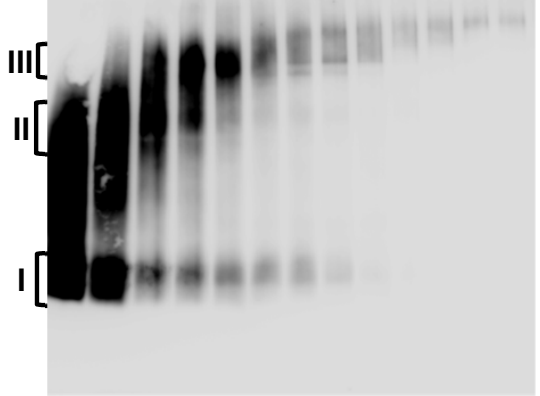

GRBC1/2 (RESC)

$3 \quad 4 \quad 5 \quad 6 \quad 78 \quad 9101112131415$

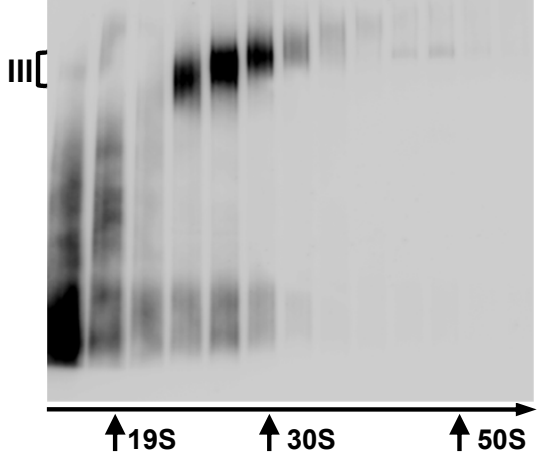

3' A-tailing

3' A/U-tailing

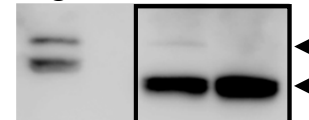

४KPAF4-TAP ४KPAF4-CBP
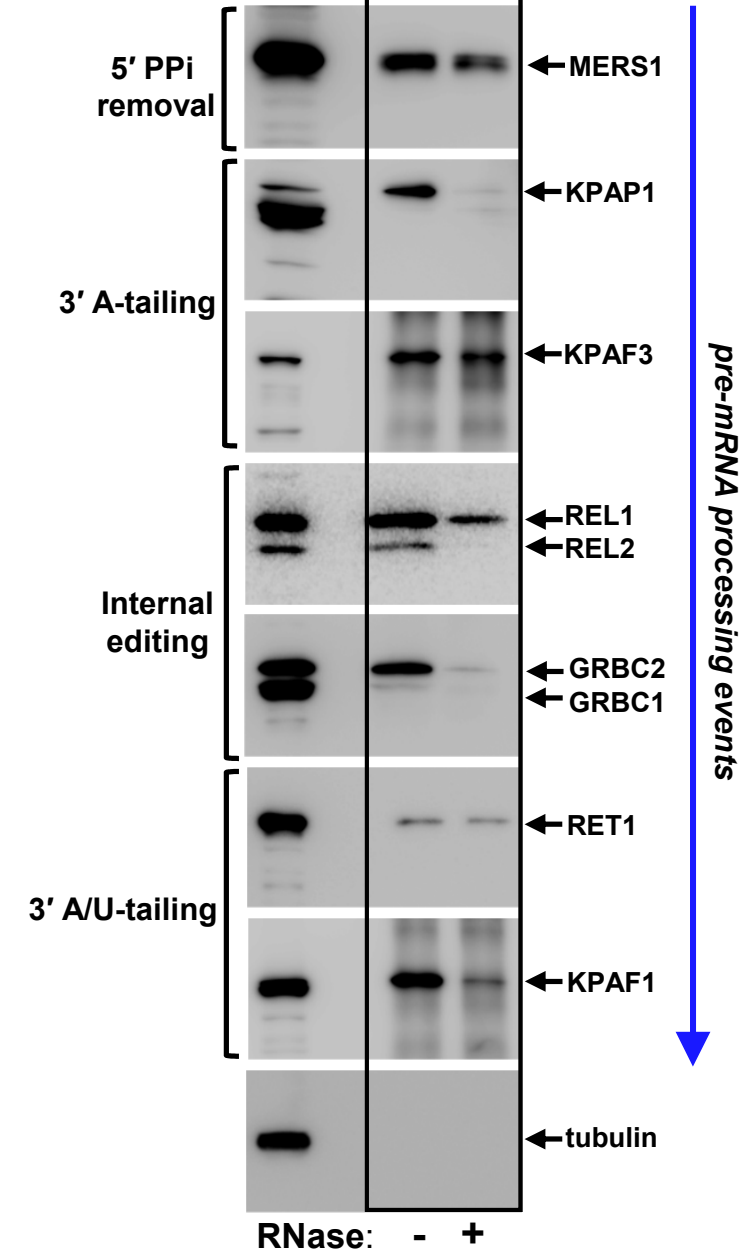

KPAF4-TAP (RNase)

$\begin{array}{llllllll}3 & 4 & 5 & 6 & 7 & 8 & 9 & 101112131415\end{array}$

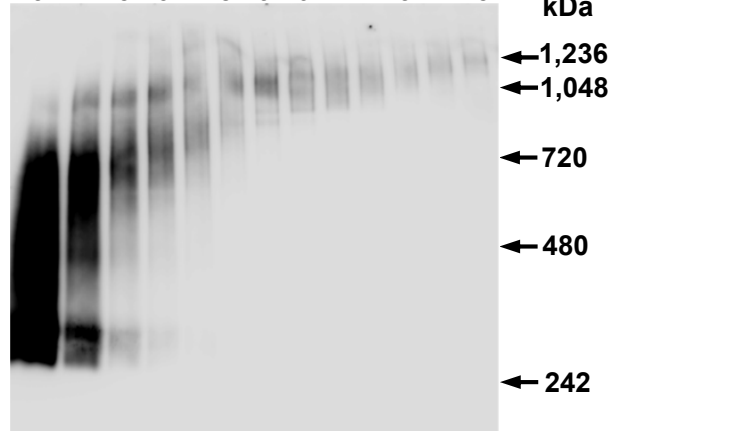

REL1/2 (RECC)

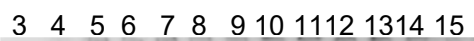

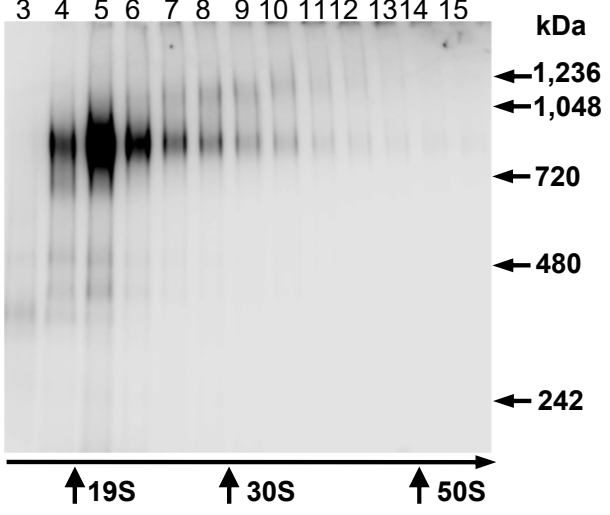

Fig. 1. 
bioRxiv preprint doi: https://doi.org/10.1101/456418; this version posted October 29, 2018. The copyright holder for this preprint (which was not certified by peer review) is the author/funder. All rights reserved. No reuse allowed without permission.

a

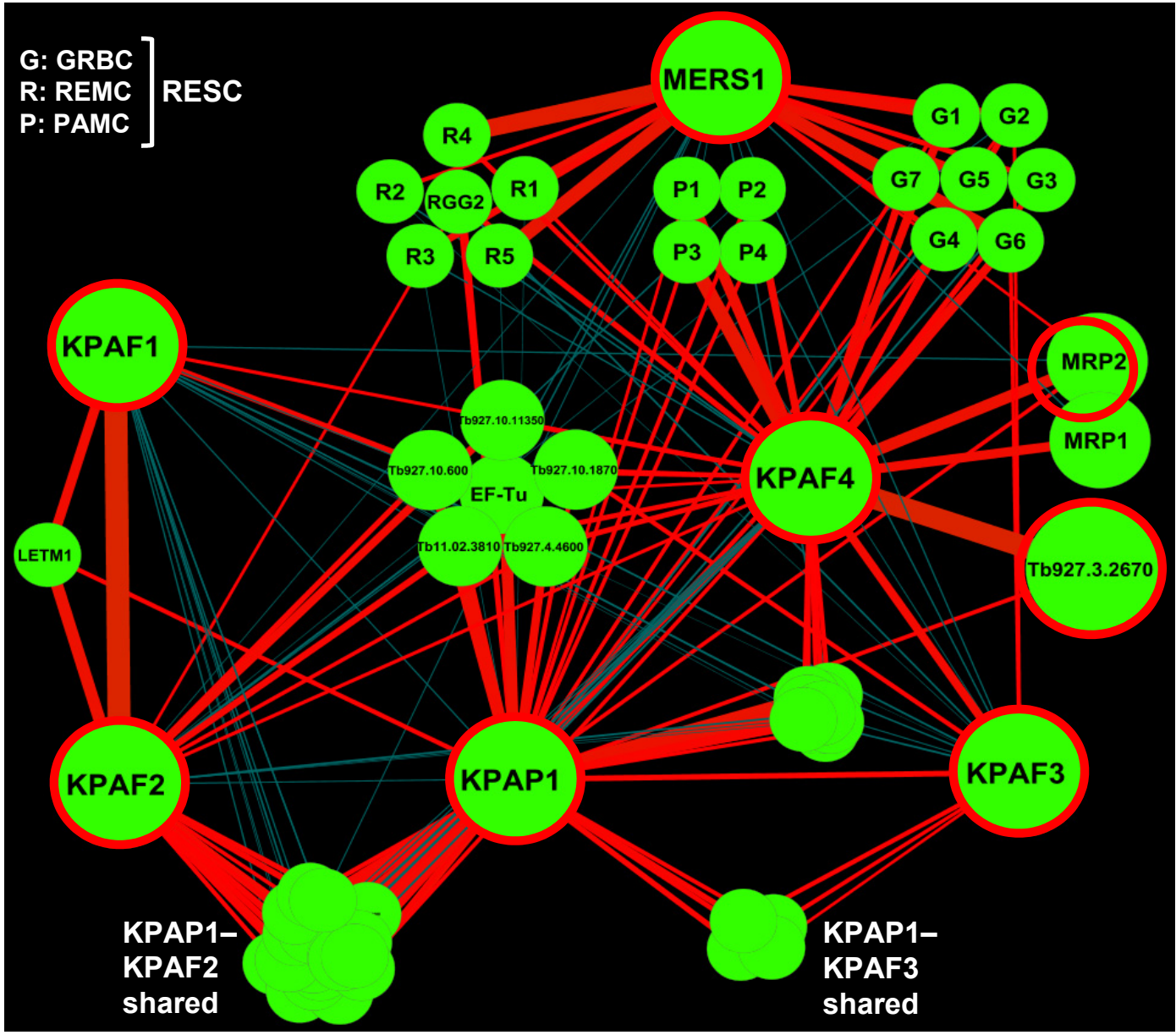

b

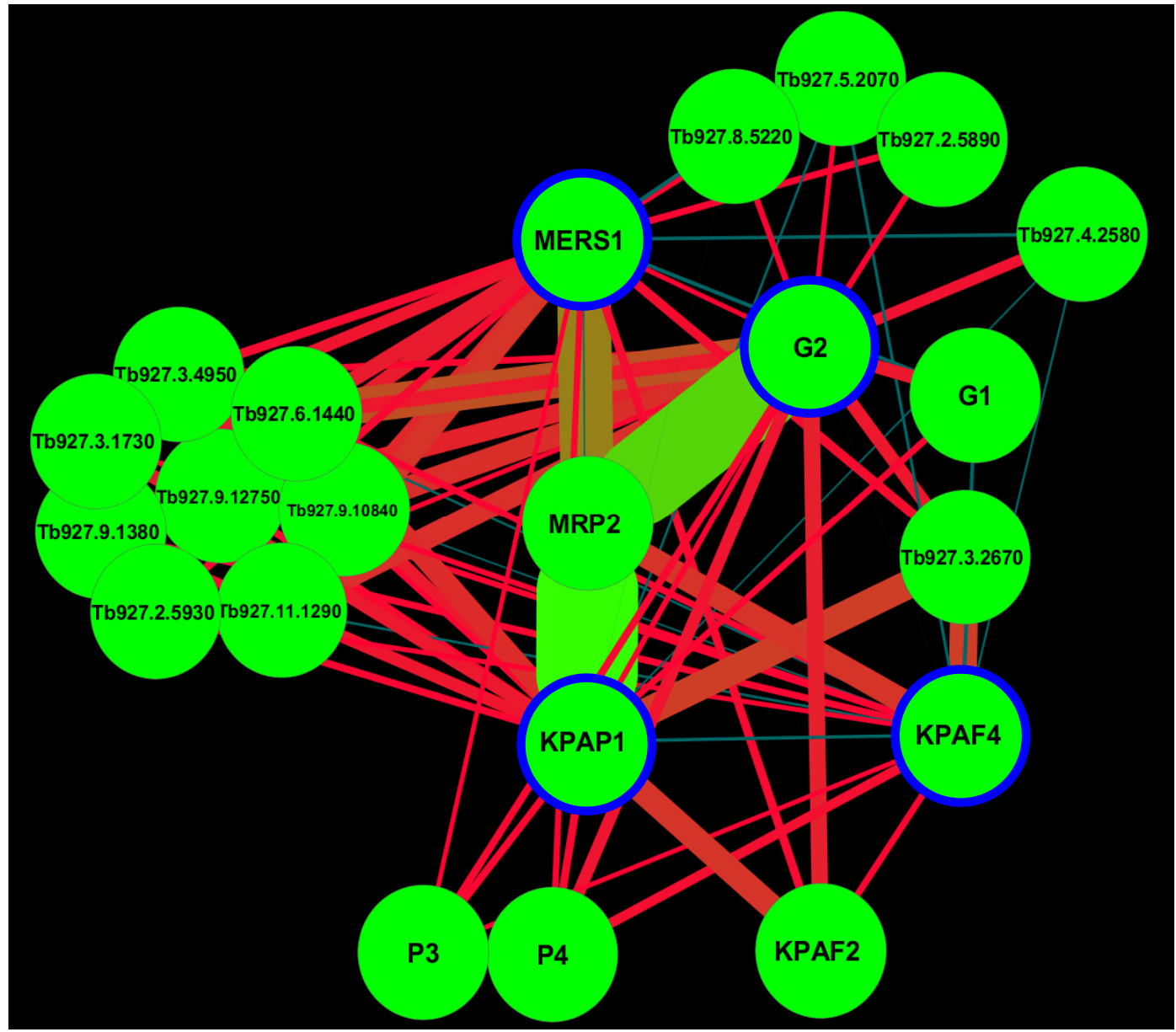

Fig. 2. 
a

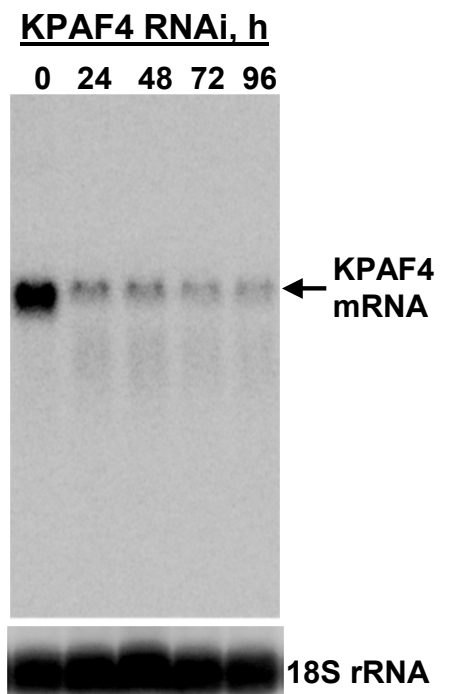

18S rRNA

C

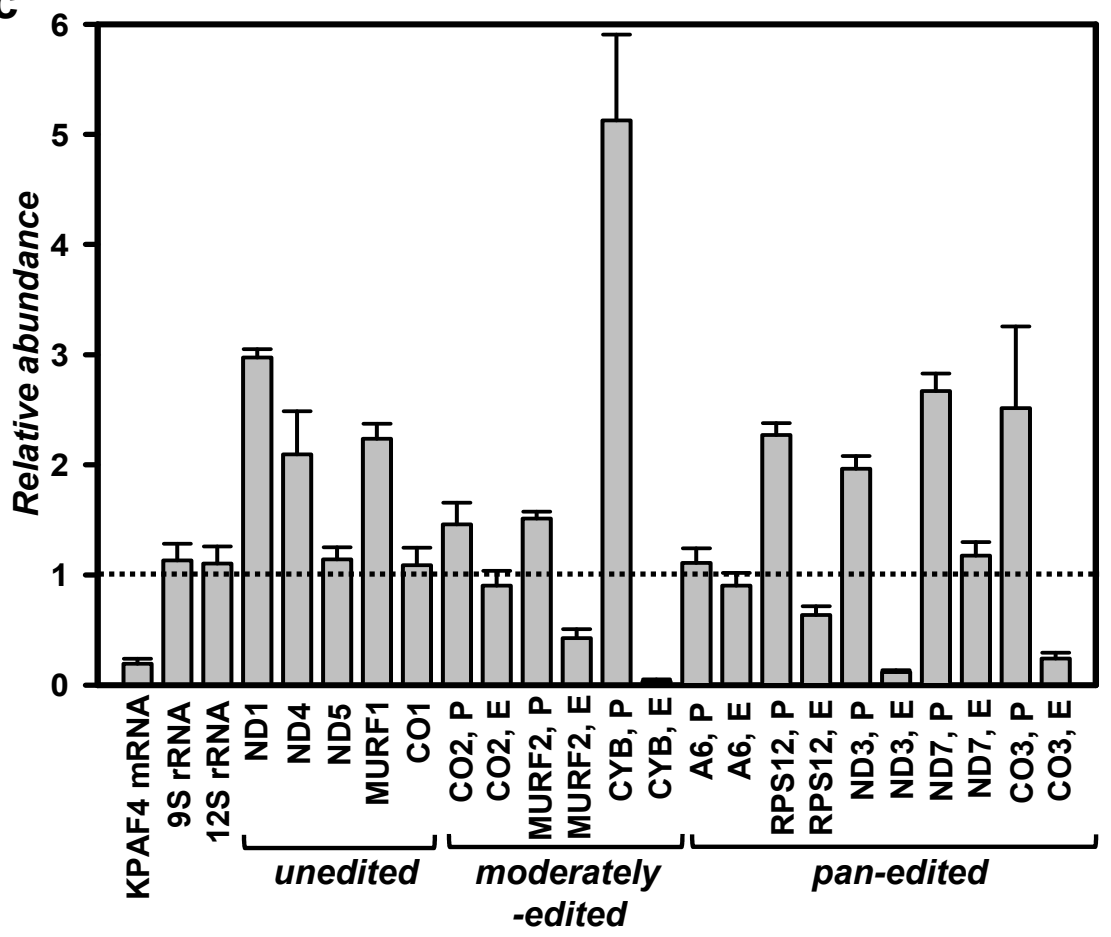

b

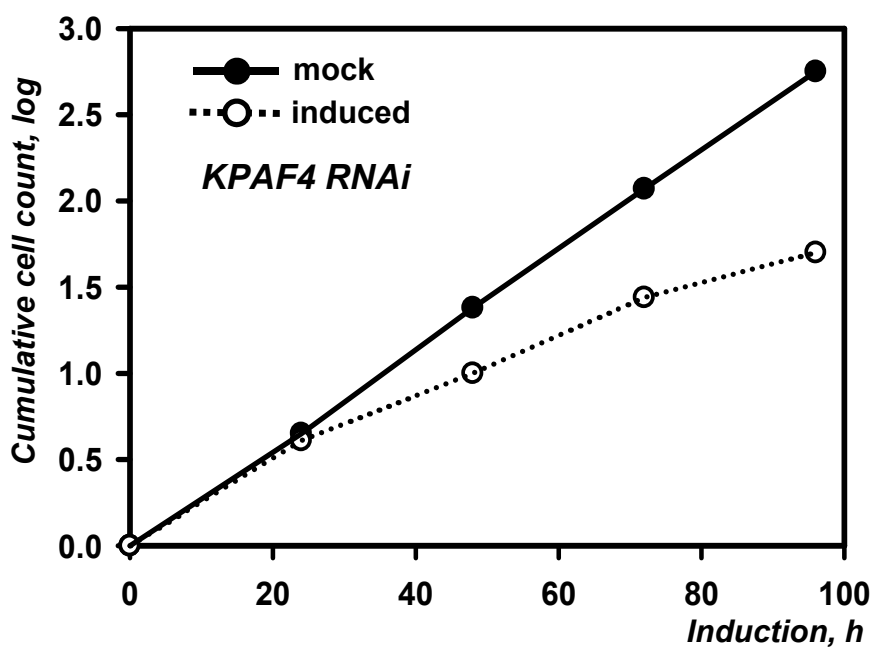

d KPAF4 RNAi, $h$

$\begin{array}{lllll}0 & 24 & 48 & 72 & 96\end{array}$

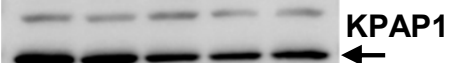

\%: $100 \quad 84 \quad 56 \quad 49 \quad 54$

KPAF1

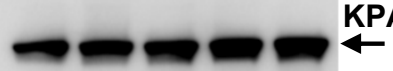

\%: $100 \quad 90 \quad 94 \quad 119 \quad 110$

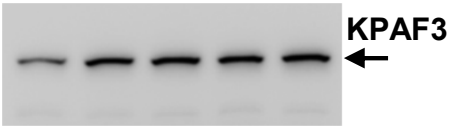

\%: 1001059611198

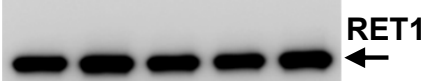

$\%: 1001109697 \quad 106$

GRBC2

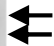

\%: $10010194 \quad 97102$

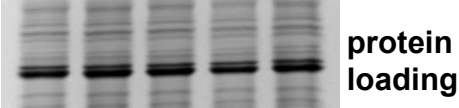
loading 


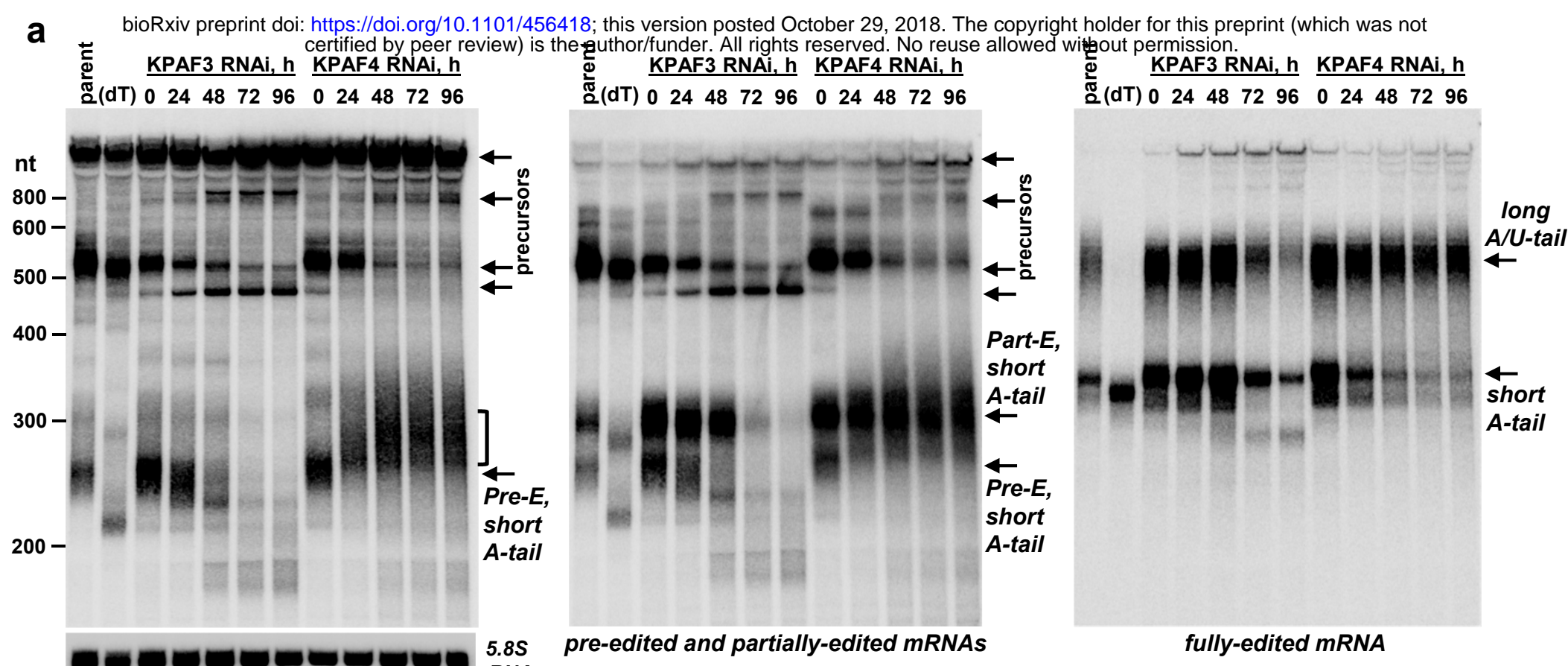

\section{pre-edited mRNA} rRNA


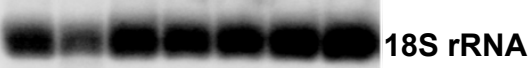

d

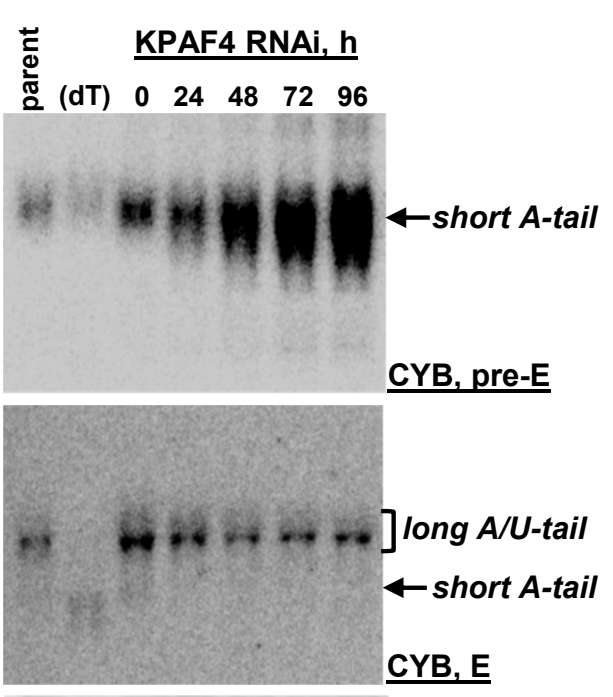

b

e
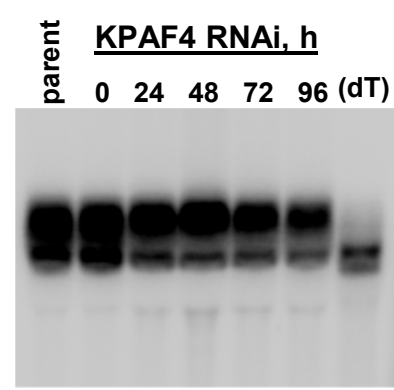

C01, unedited

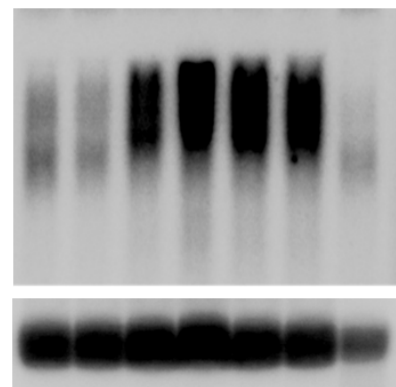

]long A/U-tail

$\leftarrow$ short A-tail

ND1, unedited

18S rRNA f

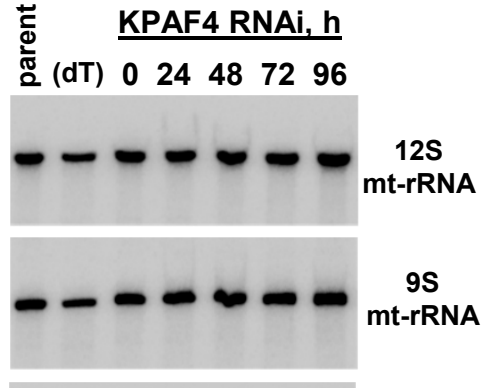

$\omega \sigma \infty \omega 0$ 5.8S rRNA

\% $\begin{array}{lllll}0 & 24 & 48 & 72 \quad 96\end{array}$

a - a

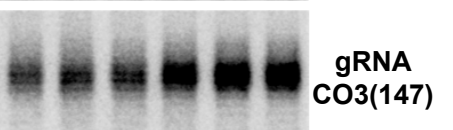

18S rRNA

Fig. 4. 


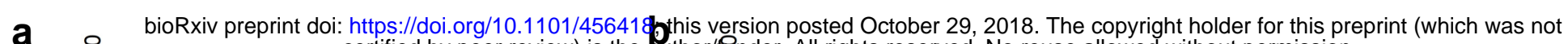

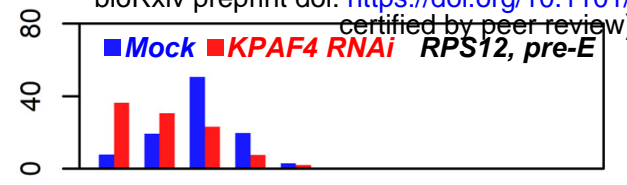



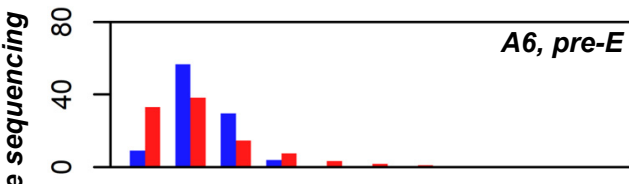

80

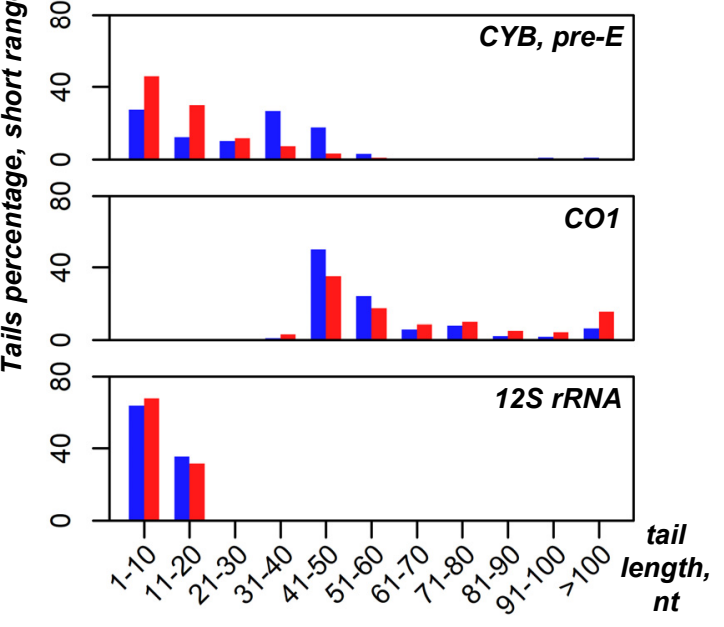

C
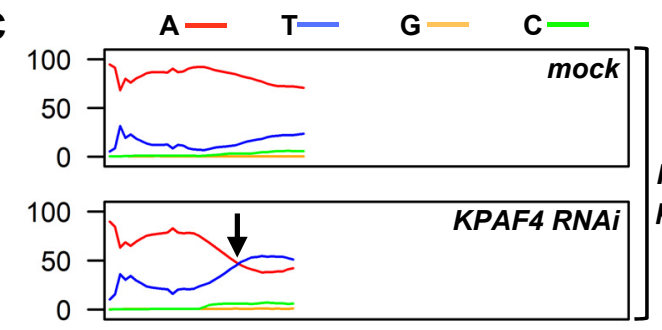

RPS12,

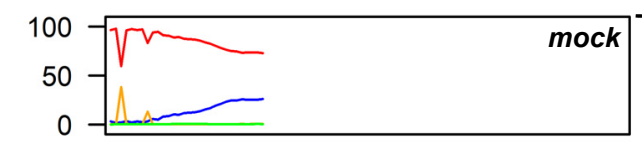

ड़
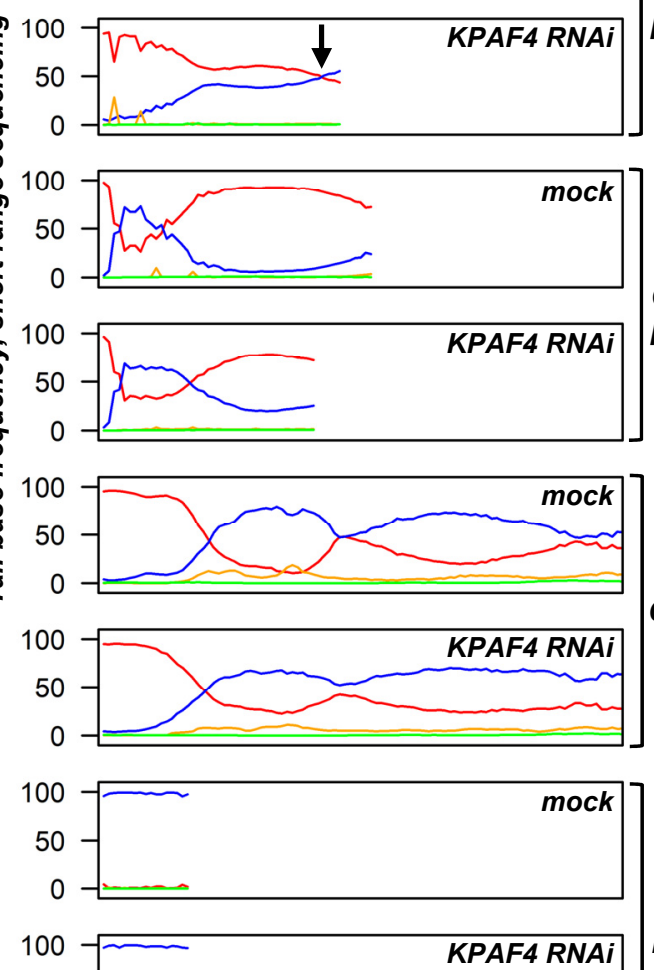

50

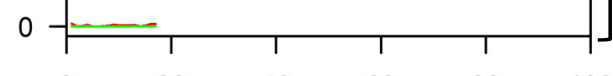

$\begin{array}{lllll}20 & 40 & 60 & 80 & 100\end{array}$

Distance from encoded 3' end, nt pre-E
CYB,

pre-E

A6, pre-E C01

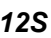

rRNA

(1) allowed without permission.

RPS12, E
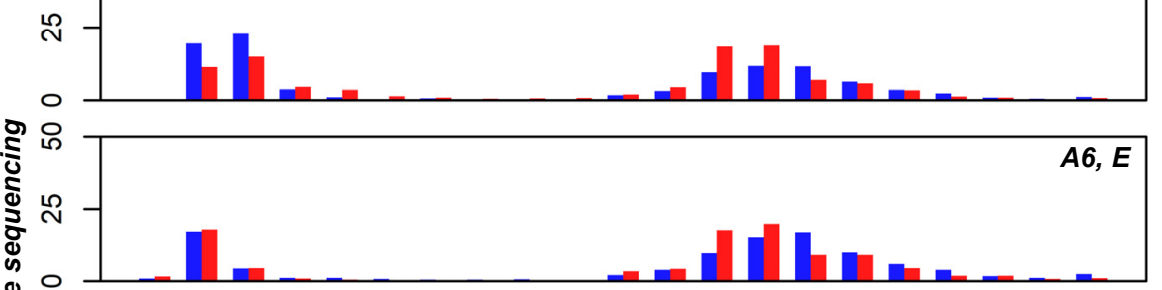

$\stackrel{0}{0} 0$

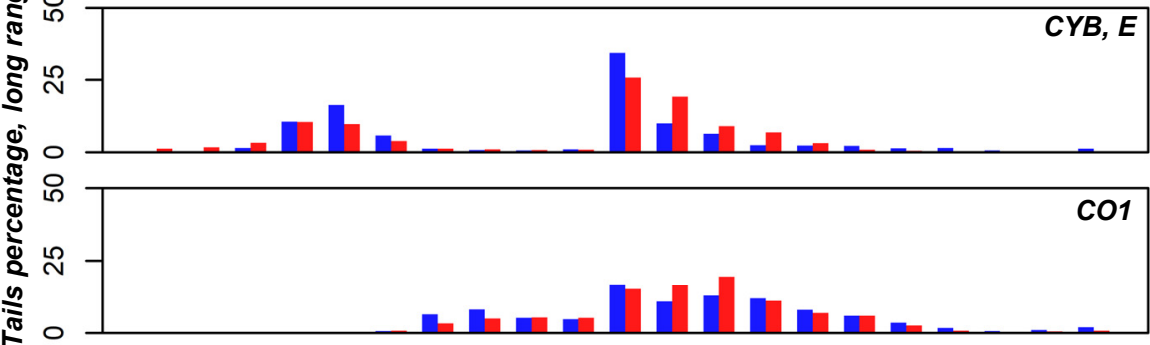

○

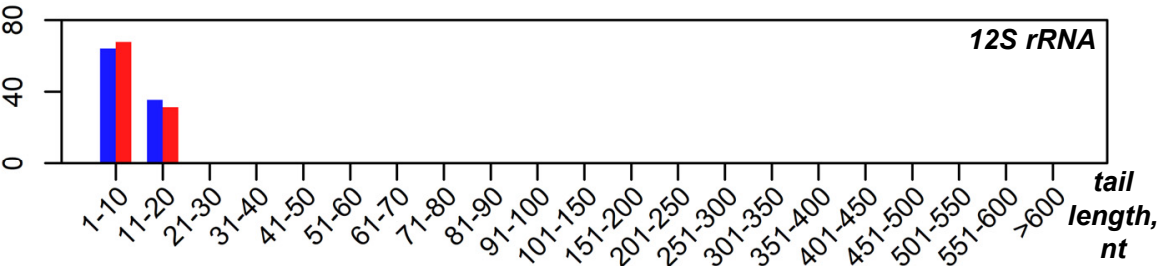

d

$$
\mathbf{A}-\mathbf{T}-\mathbf{G}-\mathbf{C}-
$$
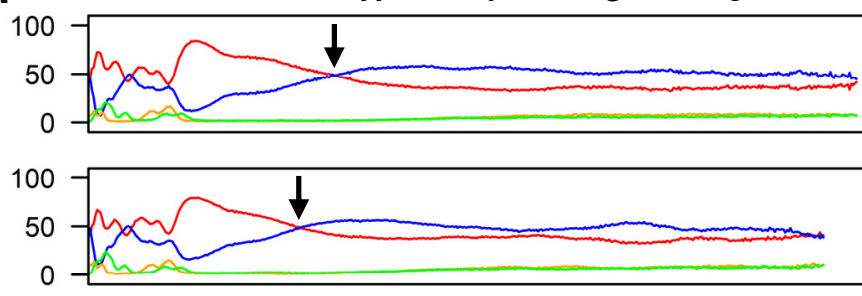

KPAF4 RNAi

RPS12,

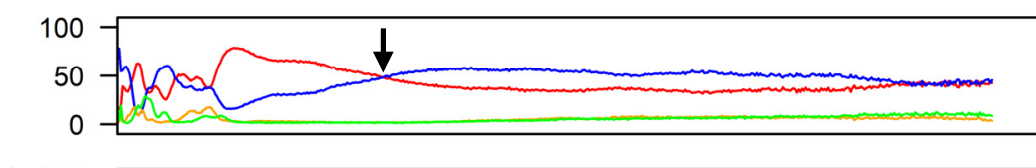

:

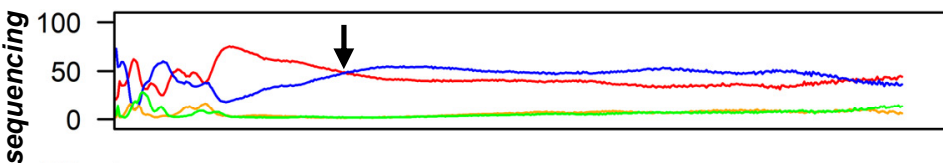

KPAF4 RNAi

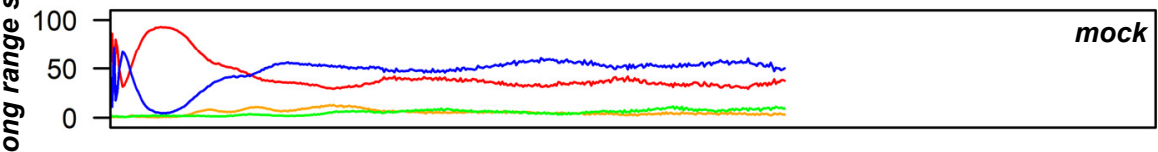

ปั

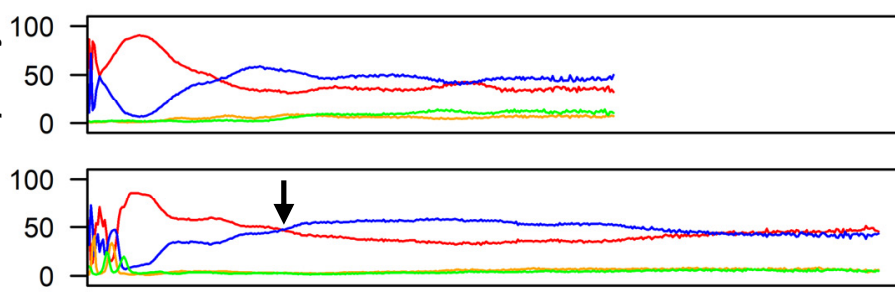

KPAF4 RNAi

CYB,

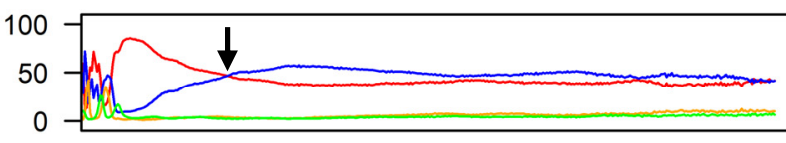

KPAF4 RNAi

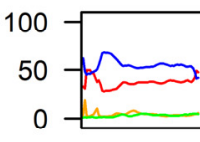

mock

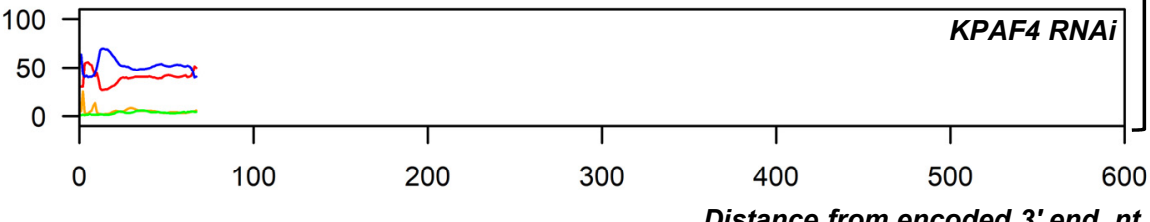

$12 S$ rRNA 
a bioRxiv preprint doi: https://doi. bg/10.1101+456418; this version posted Oetober 29, 2018. The eopyright holder for this preprint (which was not
certified by peer review) is the author/funder. All rights reserved. No reuse allowed without permission.
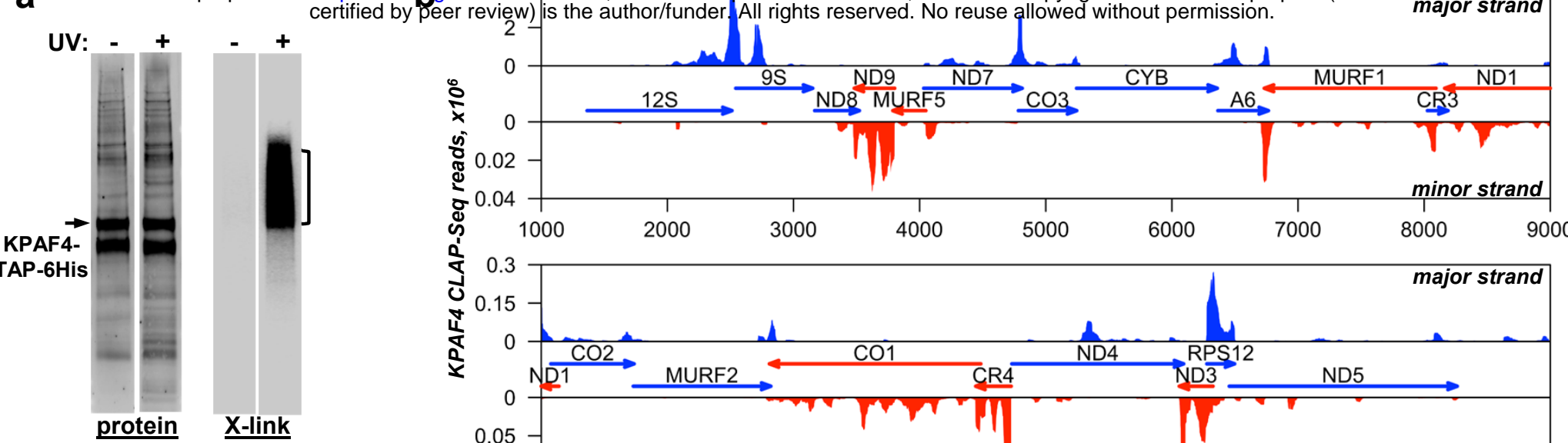

protein

$\underline{X-l i n k}$

过 0.3

C

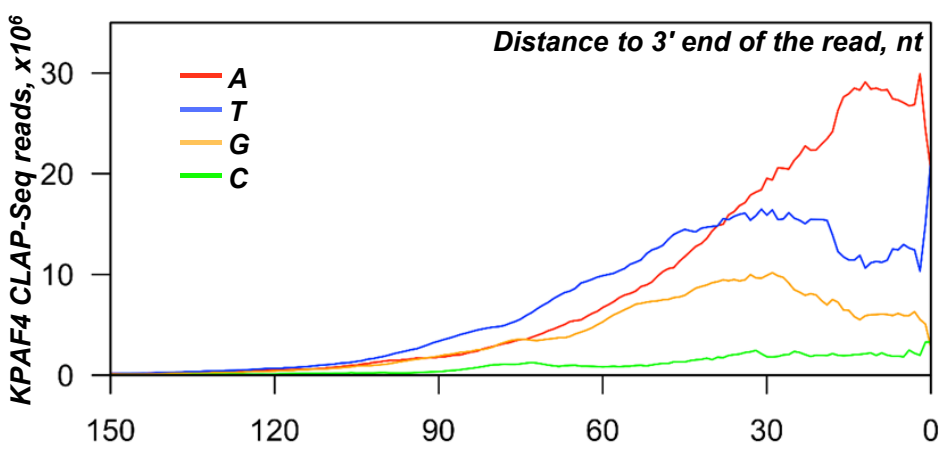

e
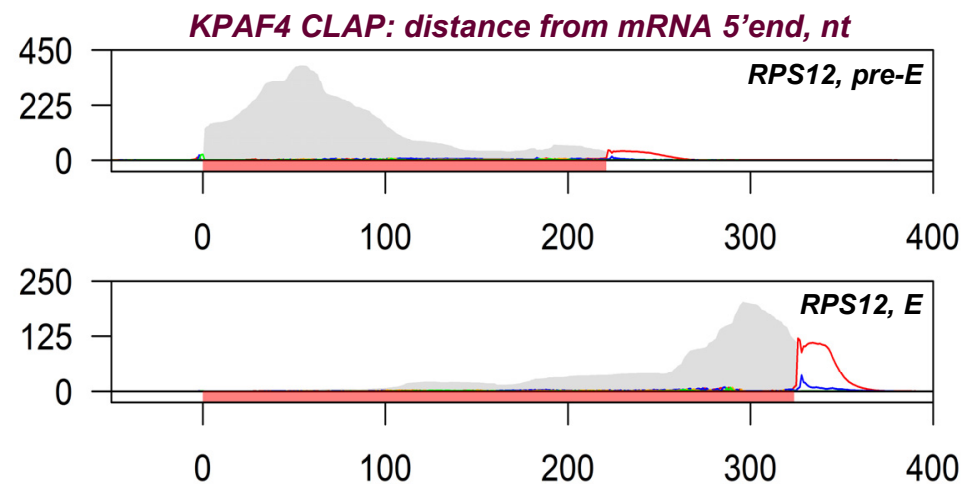

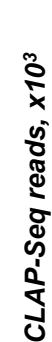$$
\text { (1) }
$$

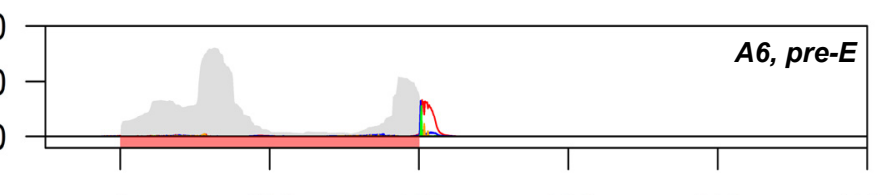

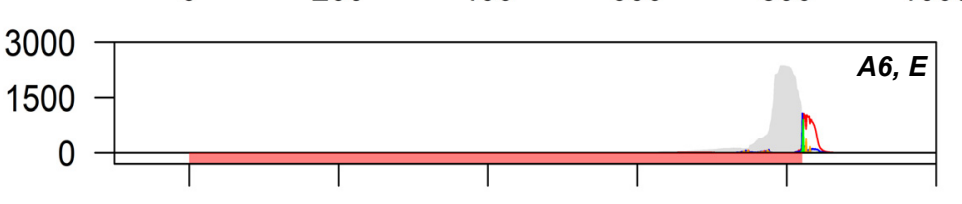
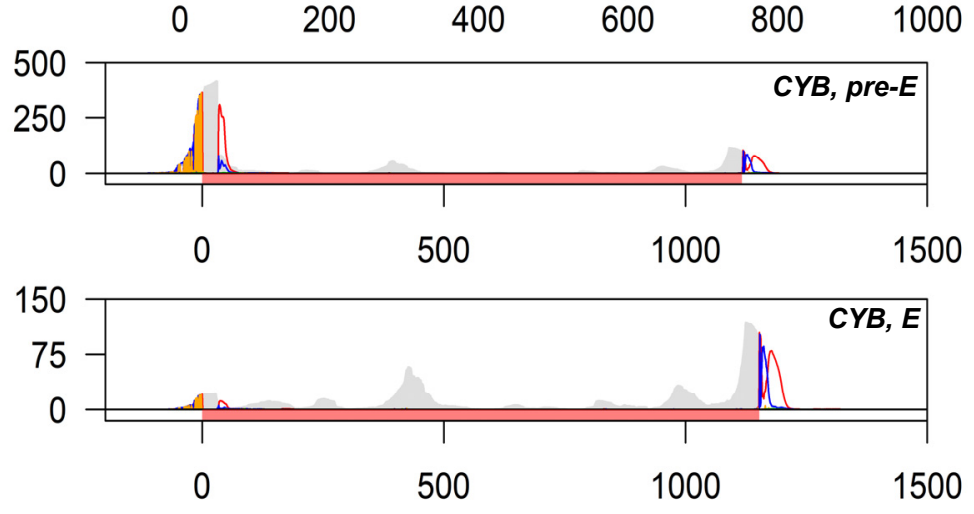

d

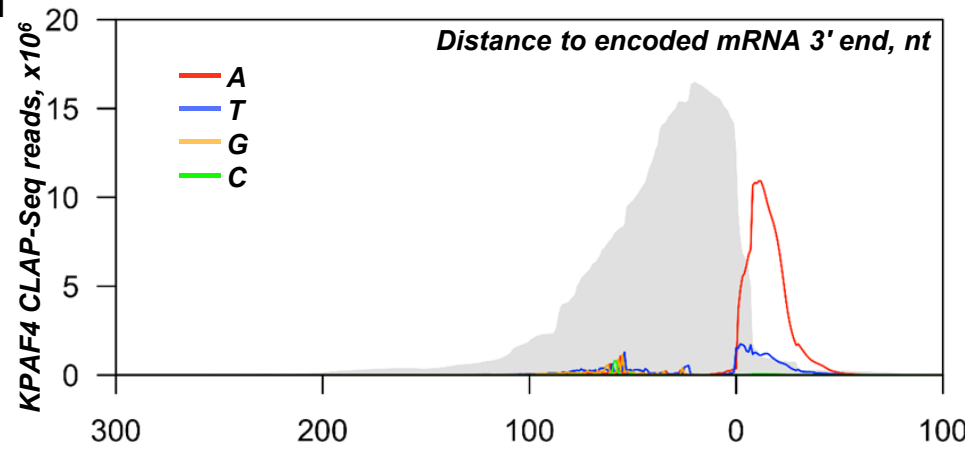

f
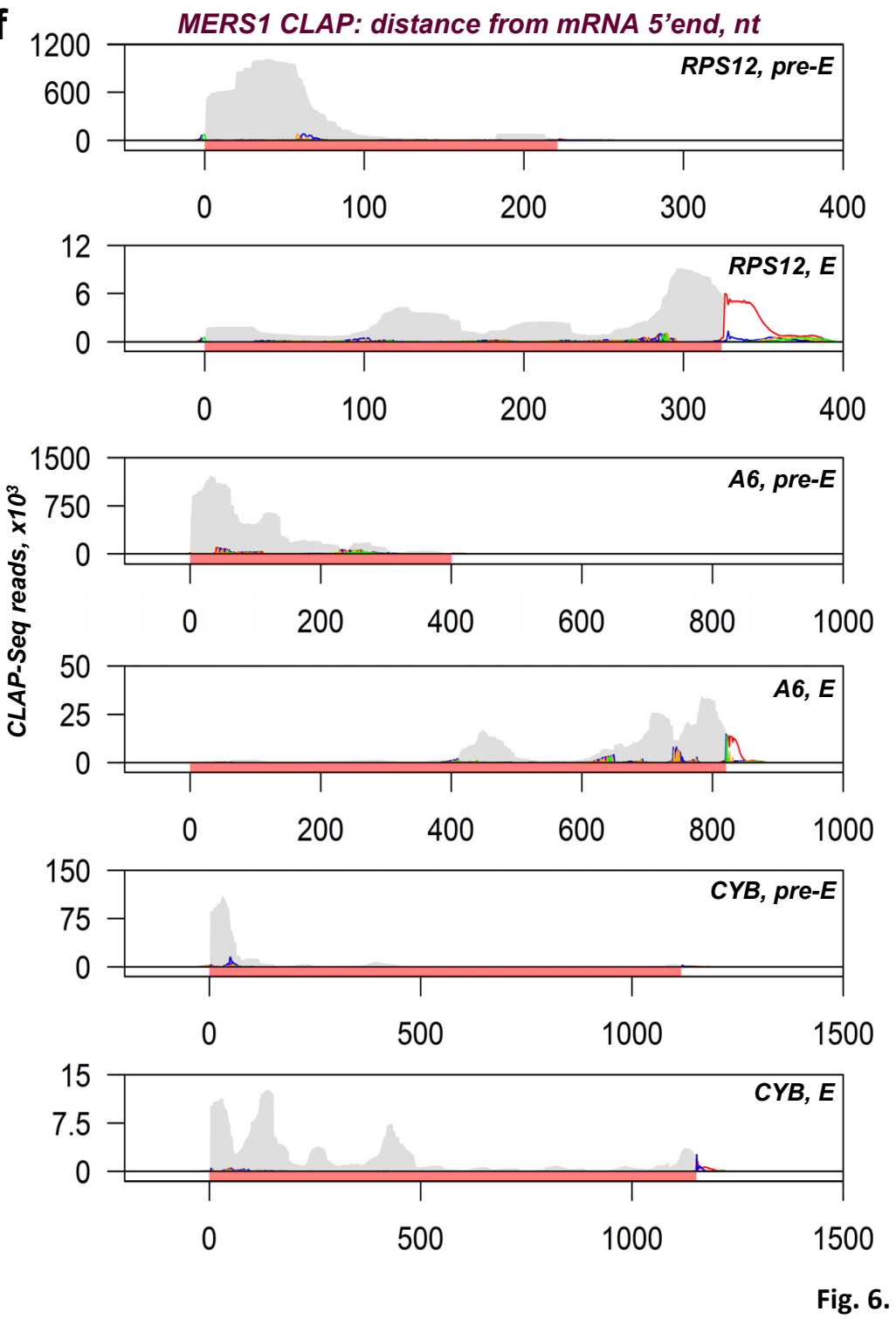
bioRxiv preprint doi: https://doi.org/10.1101/456418; this version posted October 29, 2018. The copyright holder for this preprint (which was not

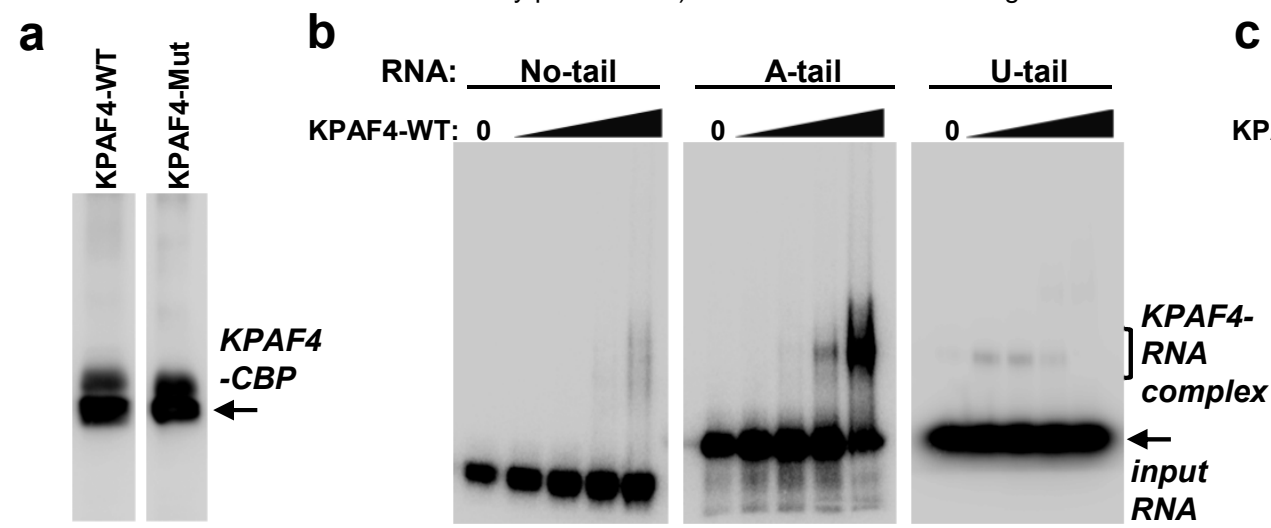

KPAF4 complexes, Western blot

C

EMSA, KPAF4-WT: 0, 5, 15, 50 and $150 \mathrm{nM}$
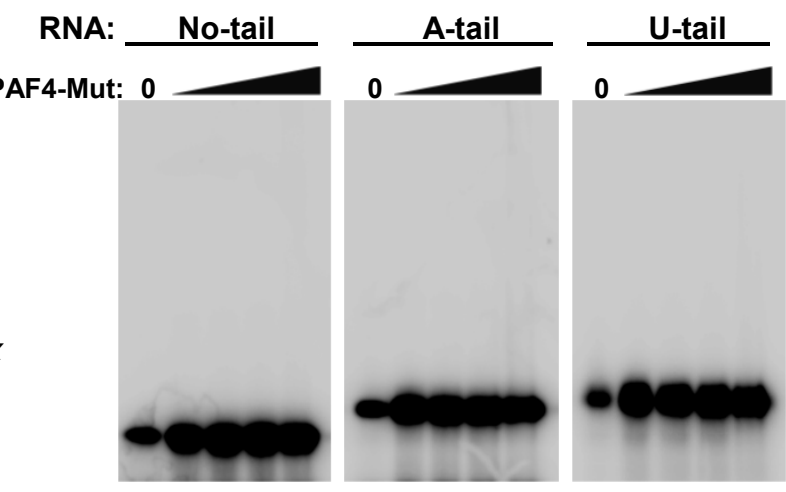

EMSA, KPAF4-Mut: $0,5,15,50$ and 150 nM d

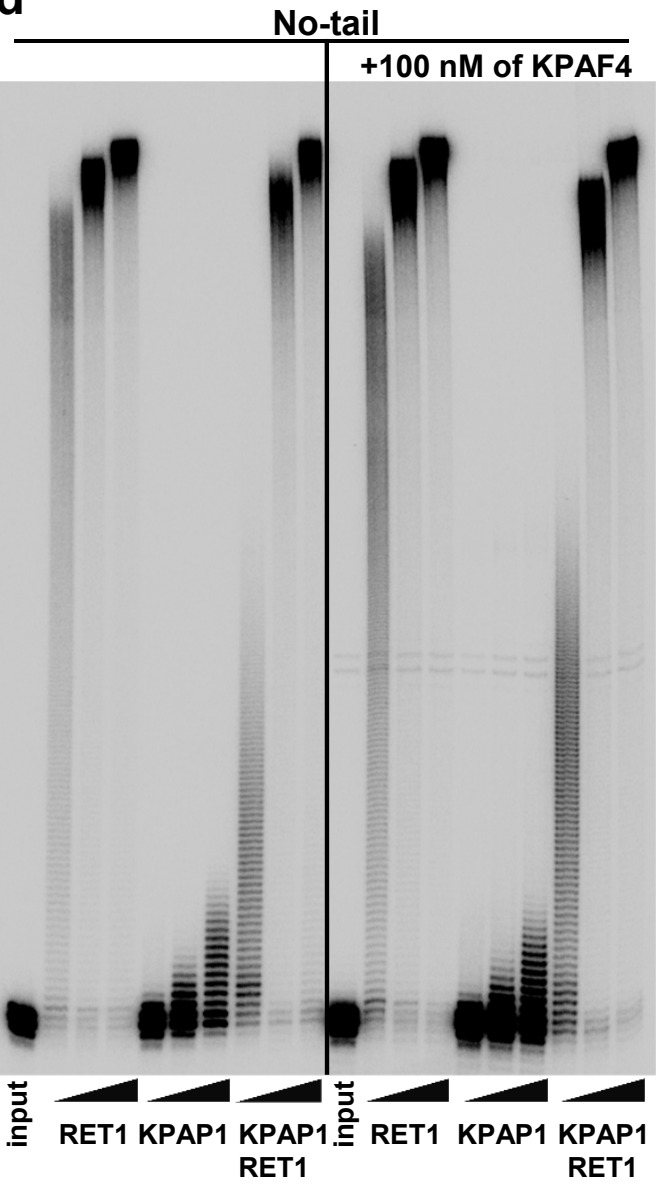

Time: 5, 10, $20 \mathrm{~min}$.


e



Time: 20 min. KPAF4: 0, 25, 50 and 100 nM 


\section{a}

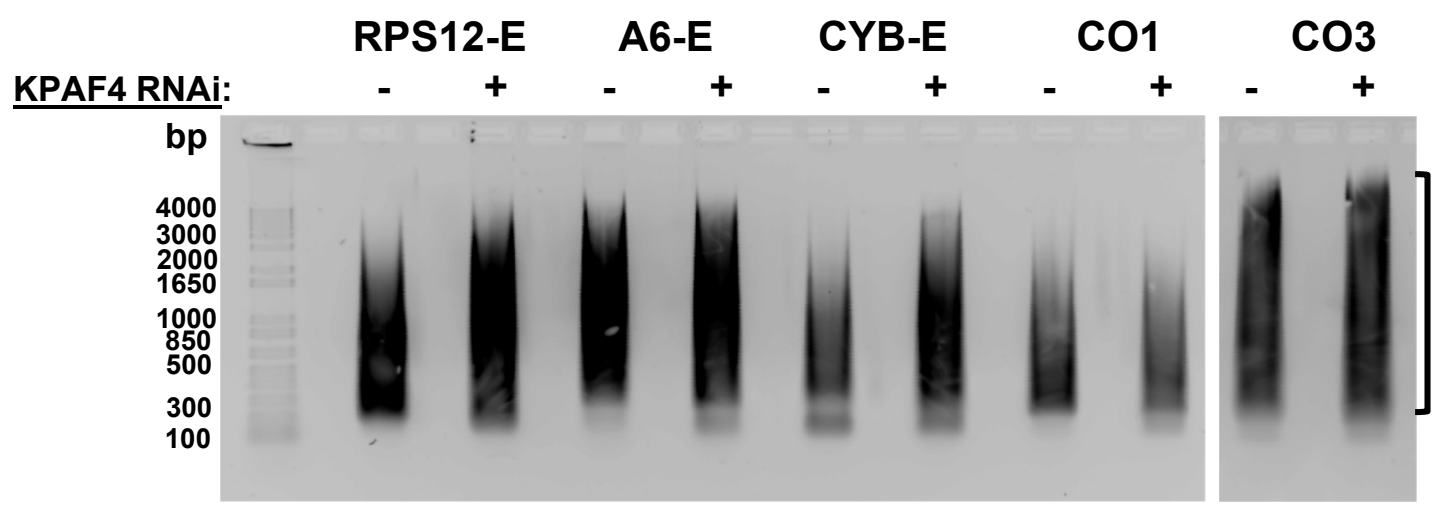

b

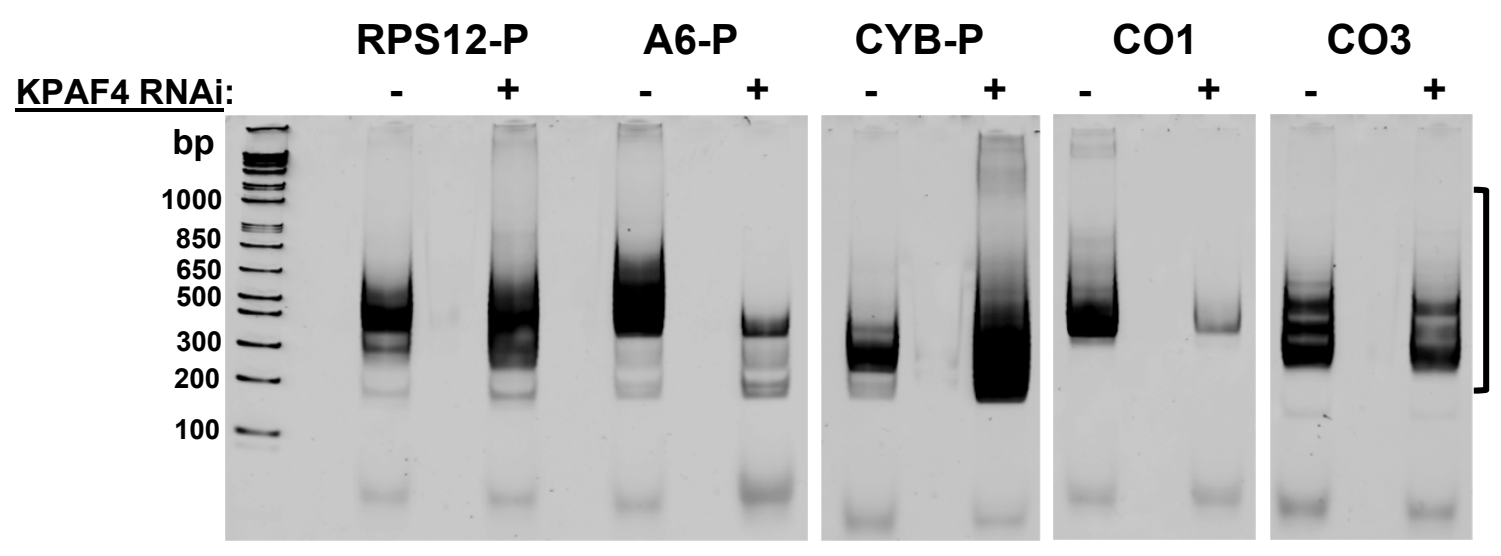

Supplementary Figure 2. Circular RT-PCR libraries for 3' extension analysis.

DNA was extracted from regions indicated by brackets. A. SMRT libraries were purified by electrophoresis in $1.2 \%$ agarose gel. B. RNA-Seq libraries were purified by electrophoresis in 5\% polyacrylamide gel. 


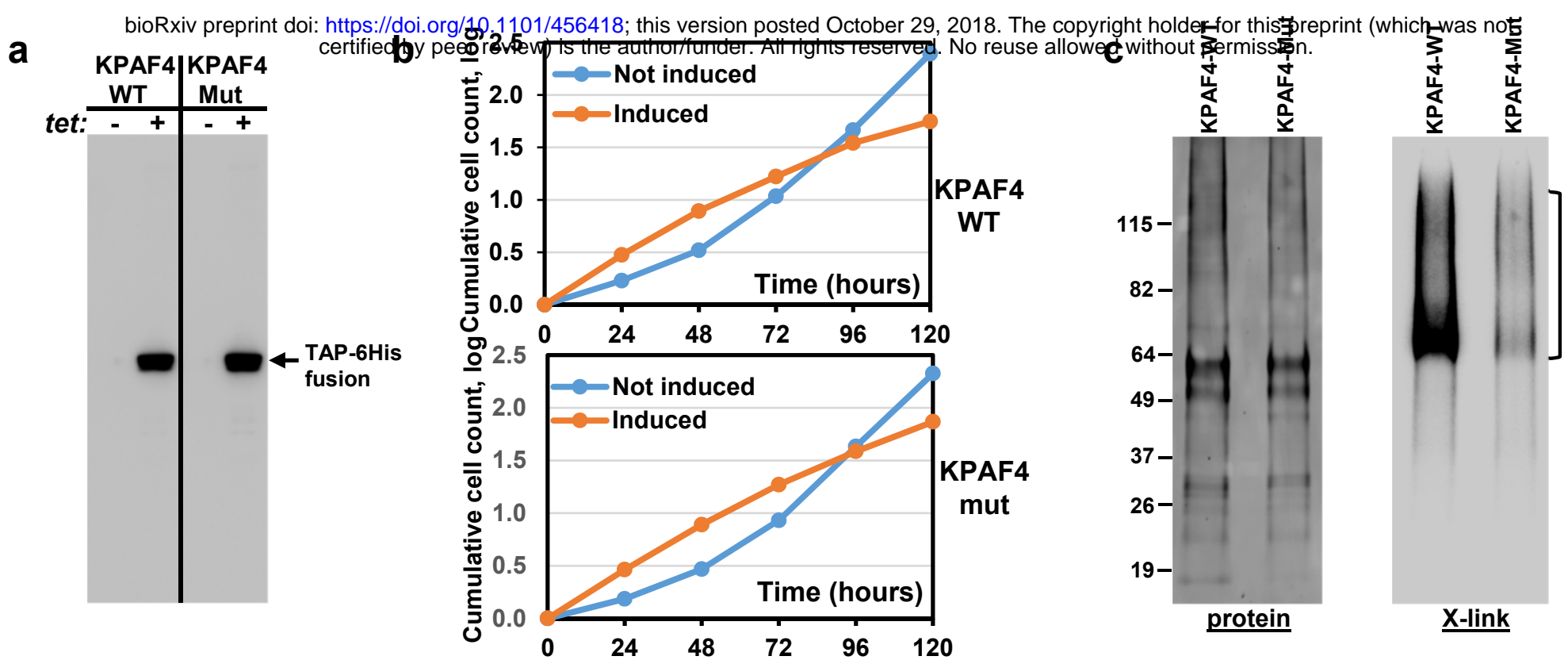

d
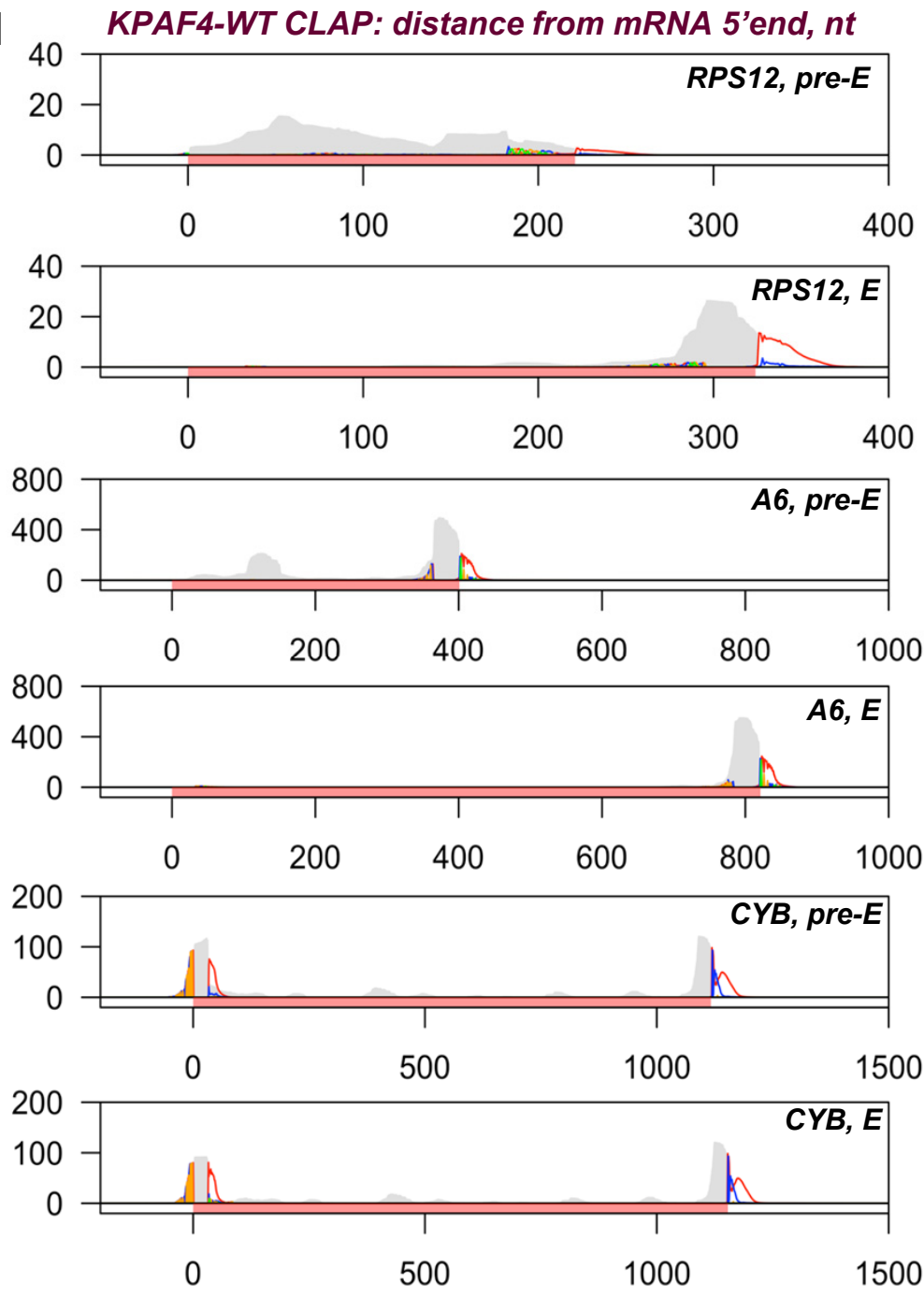

KPAF4-Mut CLAP: distance from mRNA 5'end, nt
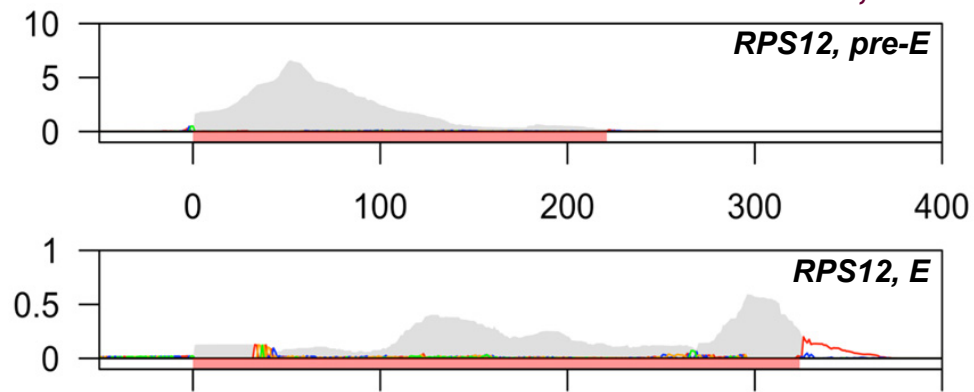

0

100

200

300

400
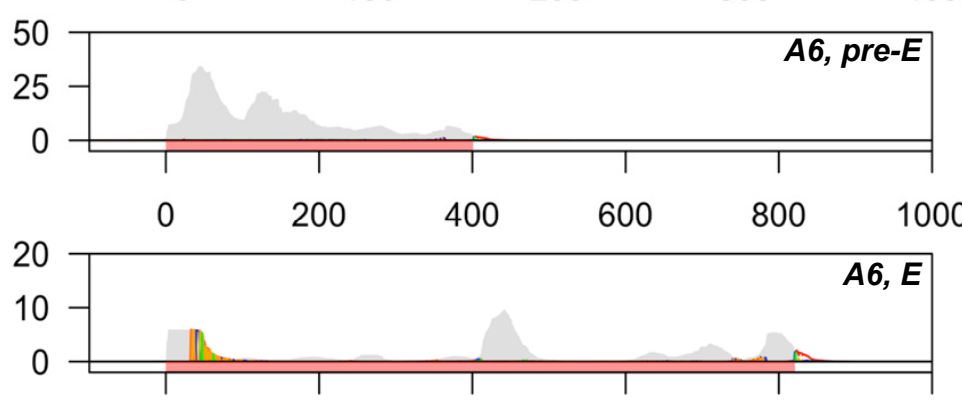

$\begin{array}{llllll}0 & 200 & 400 & 600 & 800 & 1000\end{array}$
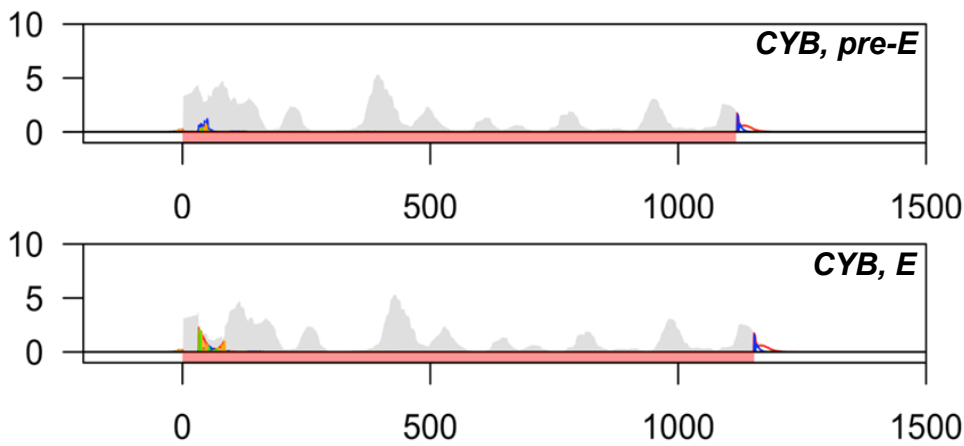

Supplementary Figure 3. KPAF4-Mut expression and in vivo binding sites analysis. (a). Inducible KPAF4-WT and KPAF4-Mut expression. Cell lysates were analyzed by western blotting with anti-CBP antibody. (b). Parasite growth kinetics after KPAF4-WT and KPAF4-Mut expression. (c) Isolation of in vivo KPAF4-RNA crosslinks. Sequenced area is indicated by brackets. (d) Crosslinked fragments were mapped to representative mitochondrial mRNAs. 

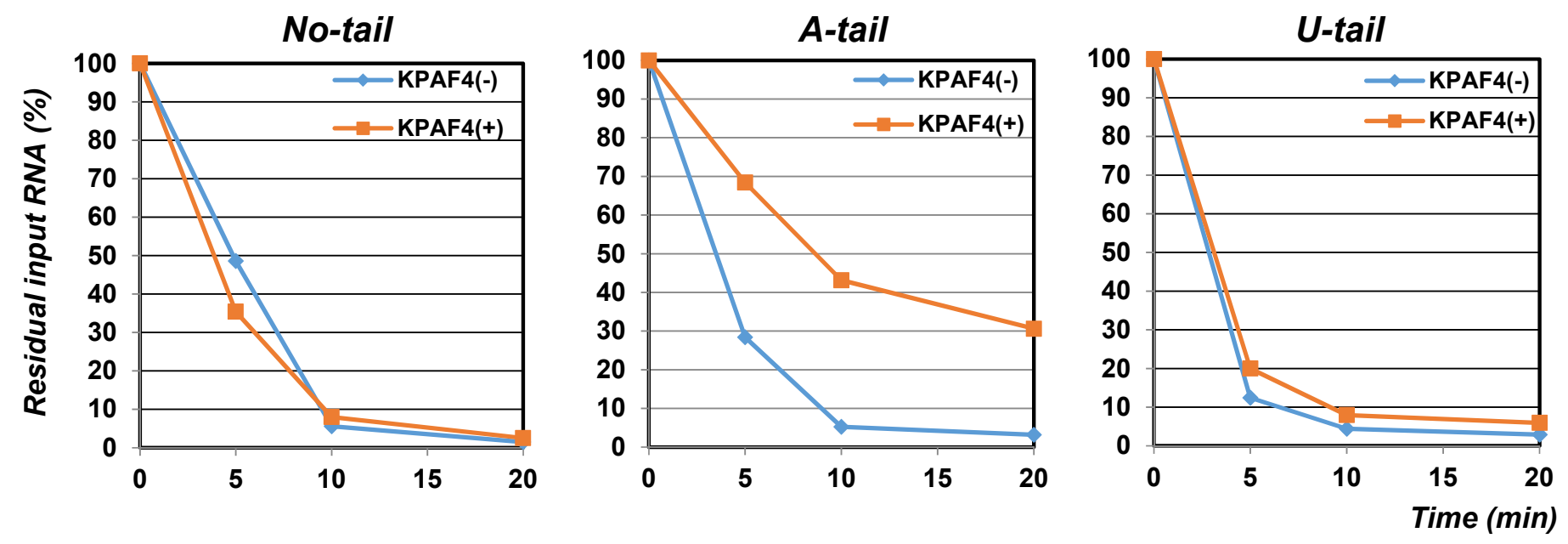

Supplementary Figure 4. Quantitation of input RNA decay in Fig. 7E. Non-saturated signals were acquired with phosphor storage screen and quantitated vs. input RNAs. 